\title{
Antibiotics in critically ill patients: a systematic review of the pharmacokinetics of $\beta$-lactams
}

Joao Gonçalves-Pereira ${ }^{1,2^{*}}$ and Pedro Póvoa ${ }^{1,2}$

\begin{abstract}
Introduction: Several reports have shown marked heterogeneity of antibiotic pharmacokinetics (PK) in patients admitted to ICUs, which might potentially affect outcomes. Therefore, the pharmacodynamic (PD) parameter of the efficacy of $\beta$-lactam antibiotics, that is, the time that its concentration is above the bacteria minimal inhibitory concentration $(T>M I C)$, cannot be safely extrapolated from data derived from the PK of healthy volunteers.

Methods: We performed a full review of published studies addressing the PK of intravenous $\beta$-lactam antibiotics given to infected ICU patients. Study selection comprised a comprehensive bibliographic search of the PubMed database and bibliographic references in relevant reviews from January 1966 to December 2010. We selected only English-language articles reporting studies addressing $\beta$-lactam antibiotics that had been described in at least five previously published studies. Studies of the PK of patients undergoing renal replacement therapy were excluded.

Results: A total of 57 studies addressing six different $\beta$-lactam antibiotics (meropenem, imipenem, piperacillin, cefpirome, cefepime and ceftazidime) were selected. Significant PK heterogeneity was noted, with a broad, more than twofold variation both of volume of distribution and of drug clearance $(\mathrm{Cl})$. The correlation of antibiotic $\mathrm{Cl}$ with creatinine clearance was usually reported. Consequently, in ICU patients, $\beta$-lactam antibiotic half-life and T > MIC were virtually unpredictable, especially in those patients with normal renal function. A better PD profile was usually obtained by prolonged or even continuous infusion. Tissue penetration was also found to be compromised in critically ill patients with septic shock.

Conclusions: The PK of $\beta$-lactam antibiotics are heterogeneous and largely unpredictable in ICU patients. Consequently, the dosing of antibiotics should be supported by PK concepts, including data derived from studies of the PK of ICU patients and therapeutic drug monitoring.
\end{abstract}

Keywords: administration, dosage, $\beta$ ? $\beta$ ?-lactam antibiotics, microdialysis, pharmacodynamics, pharmacokinetics, ICU

\section{Introduction}

Infection and sepsis, whether community- or hospitalacquired, are important causes of morbidity and mortality in ICU patients $[1,2]$. Despite all of the research, sepsis therapy continues to depend on supportive management of the different organ dysfunctions and failures and on specific therapy for infection with timely and appropriate antibiotics and/or focus control.

The $\beta$-lactam antibiotics, because of their large antimicrobial spectrum and low toxicity, are among the first-line therapies for critically ill patients, especially

\footnotetext{
* Correspondence: joaogpster@gmail.com

'Polyvalent Intensive Care Unit, São Francisco Xavier Hospital, Estrada do

Forte do Alto do Duque, 1449-005 Lisboa, Portugal

Full list of author information is available at the end of the article
}

when a Gram-negative infection is suspected. However, the efficacy of antibiotics is not easily evaluated, since the clinical response is usually unnoticeable before 48 hours of therapy [3]. Moreover, the unavailability of routine therapeutic drug monitoring for the great majority of these drugs makes it difficult to distinguish clinical failure due to underdosing from lack of in vivo organism susceptibility.

Considerable evidence demonstrates that free drug time above bacteria minimal inhibitory concentration $(f$ $\mathrm{T}>\mathrm{MIC}$ ) is the measure of drug exposure most closely linked to the ability of $\beta$-lactam antibiotics to kill the target bacteria [4]. T > MIC is dependent on the halflife $\left(T_{1 / 2}\right)$ of $\beta$-lactam antibiotics and their serum concentration.

\section{() Biomed Central}


The serum concentration of an antibiotic depends on the dose delivered, its bioavailability and its volume of distribution $\left(V_{\mathrm{d}}\right) . V_{\mathrm{d}}$ is a mathematical construct and refers to the size of a compartment necessary to account for the total amount of the drug, assuming that its concentration in the whole body is equal to that measured in plasma. Drugs that distribute essentially in the extracellular fluid (mainly hydrophilic) have low $V_{\mathrm{d}}$, whilst drugs that have rapid cellular uptake (lipophilic) have high $V_{\mathrm{d}}[5,6]$.

Both $V_{\mathrm{d}}$ and drug clearance $(\mathrm{Cl})$ may be increased in ICU patients [7]. Therapeutic procedures, notably large-volume and blood products infusions, positive pressure ventilation, surgical procedures, capillary leak and reduction in albumin serum concentration all contribute to alter the concentration-time relationship of many drugs. A rise in the $V_{\mathrm{d}}$, although it reduces drug concentration, might proportionally increase $\mathrm{T}_{1 / 2}$, since $\mathrm{T}_{1 / 2}=V_{\mathrm{d}} /(\mathrm{Cl} \times 0.693)$ [7]. On the contrary, a high $\mathrm{Cl}$ may reduce the exposure of antibiotics to bacteria (Figure 1).

Renal $\mathrm{Cl}$ may be increased in septic patients because of increased renal blood flow. This has recently been shown to be a common finding in ICU patients, particularly surgical and trauma patients [8] but also septic medical patients [9]. Besides, in the study by Baptista et al. [9], the authors showed that commonly used formulas used to calculate glomerular filtration rate usually underestimate creatinine $(\mathrm{Cr}) \mathrm{Cl}$. Consequently, these authors recommended direct $\mathrm{Cr} \mathrm{Cl}$ measurement.
Moreover in ICU patients, maldistribution of blood flow in the microcirculation [10], namely, in patients in septic shock, may further decrease the drug concentration in the infected tissue [11]. These pharmacokinetic (PK) changes are sometimes influenced by the clinical course of the infection itself [12]. Consequently, PK parameters measured in healthy volunteers may not correctly predict concentrations in septic ICU patients, particularly early in the course of a severe infection $[13,14]$.

Ideally, individualized dosing strategies should account for the altered PK and pathogen susceptibility in each patient. Despite the fact that some studies addressed this issue, this information had not yet been aggregated. Furthermore, $\beta$-lactam antibiotic PK are rarely analysed outside clinical trials. Therefore, we performed a systematic review of studies that addressed the PK parameters of $\beta$-lactam antibiotics in ICU patients to assess the relationship between dose and schedule of $\beta$-lactam antibiotics and their adequacy according to pharmacodynamic (PD) end points. We also reviewed studies assessing the concentrations of $\beta$-lactam antibiotics in different tissues. Our primary intention was to aggregate PK information in this particular population and to contribute to the design of individualized dosing regimens of these drugs.

We also included studies that involved the development of PD models using PK of ICU patients and bacterial MICs. These techniques allow the calculation of the presumed T > MIC and therefore the percentage of patients in which the antibiotic will achieve its $P D$
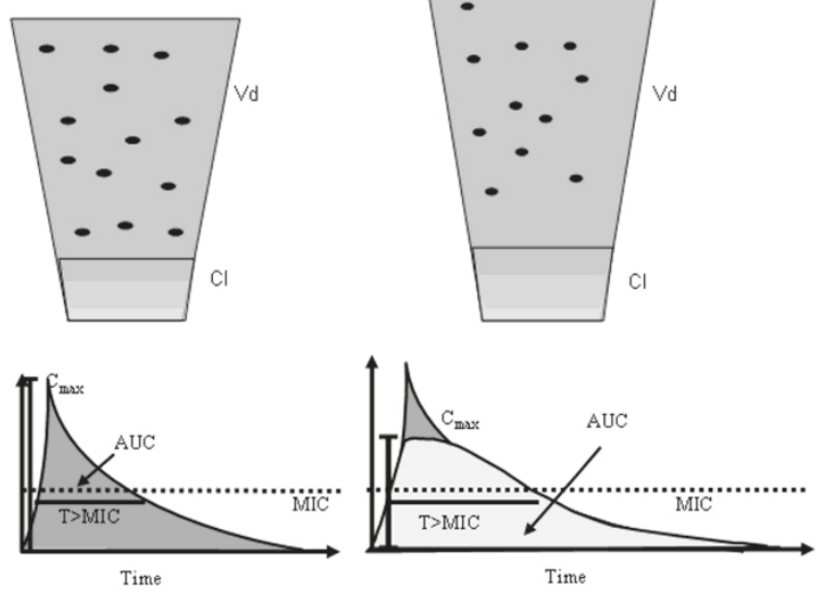

Figure 1 ICU patients present pharmacokinetic changes of antibiotics that may alter bacterial exposure. Concentration-time curve of antibiotics in healthy volunteers (left panel). A large volume of distribution $\left(V_{d}\right)$ (middle panel) is often present in ICU patients, leading to decreased maximum concentration $\left(C_{\max }\right)$ but a longer half-life $\left(T_{1 / 2}\right)$ and eventually higher time that the antibiotic concentration is above the bacteria minimum inhibitory concentration ( $\mathrm{T}>\mathrm{MIC}$ ). The antibiotic area under the concentration time curve (AUC) remains virtually the same. An increase in drug clearance (Cl) (right) is associated with decreases in AUC, $T_{1 / 2}$ and $T>$ MIC. Straight dotted lines-bacteria minimum inhibitory concentration. 
target: that is, the antibiotic's probability of target attainment (PTA) $[15,16]$. The cumulative fraction of response (CFR) is calculated by multiplying the PTA obtained for each MIC by the MIC distribution according to a microbiological database [16].

\section{Materials and methods}

The data for this review were identified by a search of PubMed (January 1966 to December 2010) as well as bibliographic references from relevant articles, including reviews on this subject and all selected studies. The search terms used were 'antibiotic' or 'carbapenem' or 'penicillins' or 'cephalosporins', and 'intensive care' or 'critically ill' or 'critical care' or 'severe sepsis' or 'septic shock', and 'pharmacokinetics' or 'pharmacodynamics'. All relevant studies in the English-language literature that described antibiotic PK in critically ill patients were assessed (Figure 2).

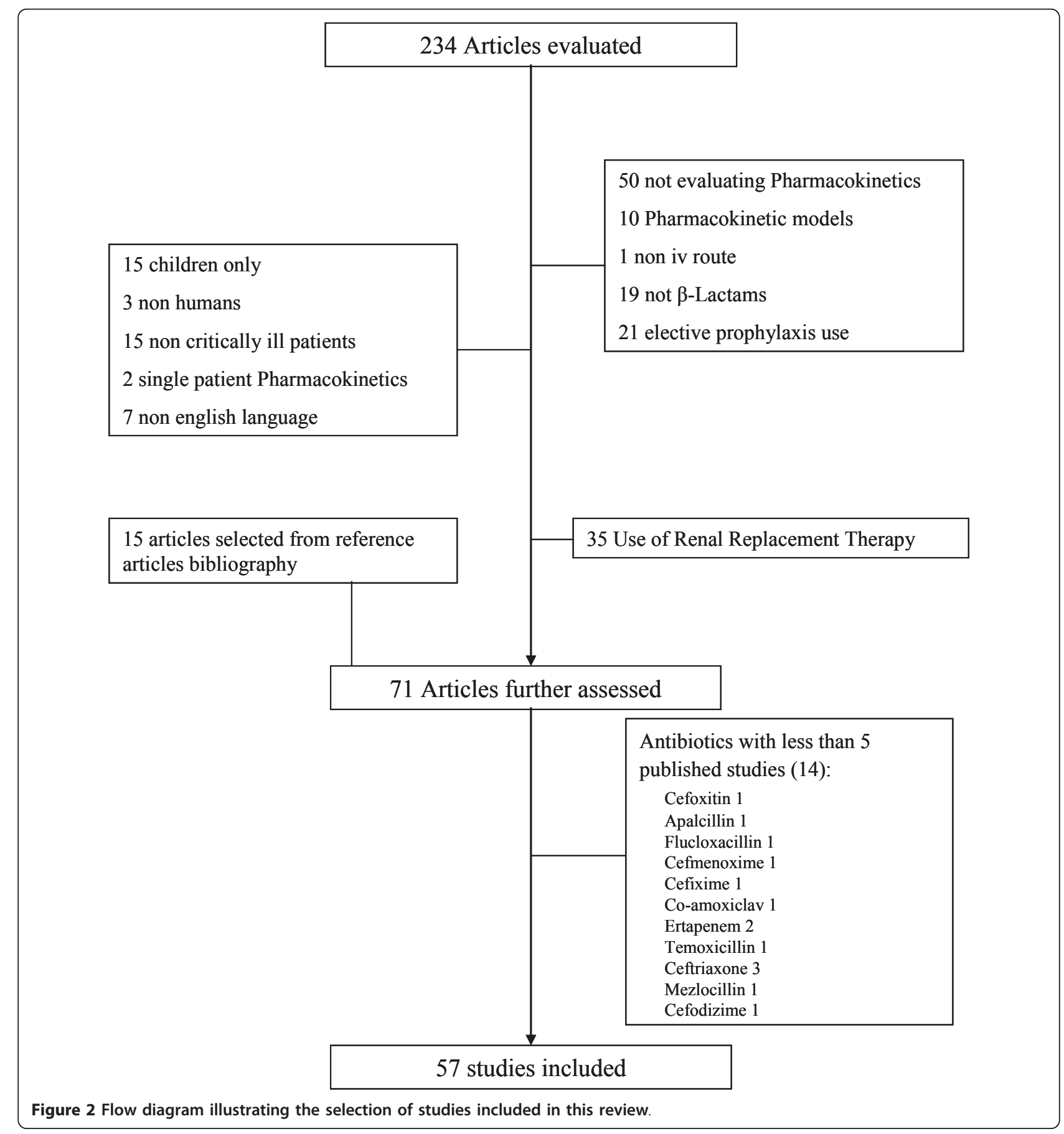


Only studies that described PK of antibiotics given intravenously to infected patients were selected. Studies referring to prophylactic antibiotics or to PK in patients under any type of renal replacement therapy were excluded. In fact, these studies are mainly directed to the measurement of $\mathrm{Cl}$ during renal replacement therapy to determine the ideal antibiotic dose and therefore are not easily compared with studies addressing the intrinsic PK of ICU patients. Furthermore, a full revision of those studies has recently been published [17].

For the purpose of our systematic review, we analysed only studies of antibiotics with at least five published references. This threshold of five referenced studies was arbitrarily chosen so that we could derive more representative and consistent data on the PK of each antibiotic. The weighted mean of the $V_{\mathrm{d}}$ was calculated so that we could present a graphic representation of each analysed antibiotic (Figure 3).

\section{Results}

A total of 57 studies assessing an aggregate of six different $\beta$-lactam antibiotics were selected.

\section{Carbapenem}

Meropenem

Several studies have addressed meropenem PK in ICU septic patients. High $V_{\mathrm{d}}$ and $\mathrm{Cl}$ have usually been reported, as well as a low binding fraction: $<10 \%$ [18]. Consequently, a large heterogeneity of PK parameters was found, exceeding a twofold variation (Table 1 and Figure 3 ). The larger reported $V_{\mathrm{d}}$, a mean of $34.4 \mathrm{~L}$, was noted on the second day of therapy in eight ventilatorassociated pneumonia (VAP) patients [19] with a mean body weight of $73 \mathrm{~kg}$. In a Thai VAP population $(N=9)$ with a lower mean body weight (only $54.2 \mathrm{~kg}$ ), the mean $V_{\mathrm{d}}$ was $6.0 \mathrm{~L}$ despite also being measured after 48 hours of therapy with meropenem [20]. This supports the hypothesis of a potential relationship between body weight and $V_{\mathrm{d}}$.

Meropenem $\mathrm{Cl}$ ranged from a mean of $4.7 \mathrm{~L} /$ hour to a mean of $15.4 \mathrm{~L} /$ hour and was generally found to be closely correlated to $\mathrm{Cr} \mathrm{Cl}$. In fact, in patients with severe sepsis, the six patients with the lower $\mathrm{Cr} \mathrm{Cl}(<50 \mathrm{~mL} /$ minute) had the higher $\mathrm{T}>\mathrm{MIC}$ and area under the concentration time curve (AUC) $(230.2 \mathrm{mg} \times$ hour $/ \mathrm{L}$ vs.

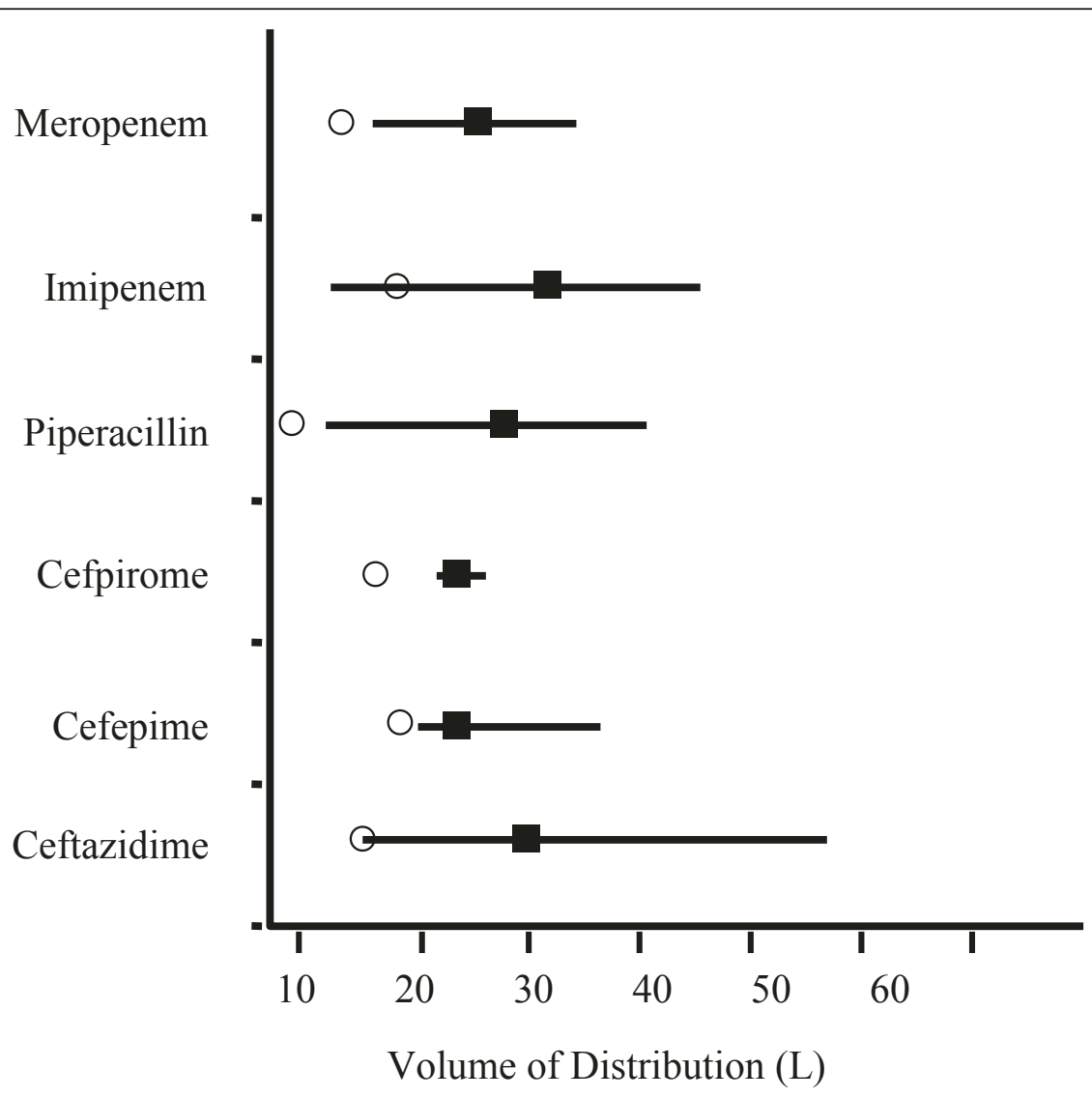

Figure 3 Heterogeneity of volume of distribution in litres of $\beta$-lactam antibiotics in ICU patients. Open circles: volume of distribution in healthy volunteers [44,51,89-92]; filled squares: weighted means of volume of distribution in the studies; straight lines: ranges of the means of volume of distribution in the studies. 
Table 1 Pharmacokinetic parameters of $\beta$-lactam antibiotics ${ }^{\mathrm{a}}$

\begin{tabular}{|c|c|c|c|c|c|c|}
\hline \multirow[b]{2}{*}{$\begin{array}{l}\text { Antibiotic drug } \\
\text { classes and drugs }\end{array}$} & \multicolumn{4}{|l|}{$\begin{array}{l}\text { PK } \\
\text { parameters }\end{array}$} & \multicolumn{2}{|l|}{ Study } \\
\hline & $V_{\mathrm{d},} \mathrm{L}$ & $\mathrm{Cl}$, L/hour & $T_{1 / 2}$, hours & Patient demographics & Study types [93] & References \\
\hline \multicolumn{7}{|l|}{ Carbapenems } \\
\hline Meropenem & $21.2 \pm 4.7^{b}$ & $11.3 \pm 4^{b}$ & $1.4 \pm 0.4^{b}$ & $\begin{array}{l}N=11 \\
\text { Age } 63.1 \text { years }[23 \text { to } \\
81] \\
\text { Mild to severe } \\
\text { intraabdominal sepsis }\end{array}$ & Descriptive & $\begin{array}{l}\text { Lovering et al., } 1995 \\
\text { [22] }\end{array}$ \\
\hline Meropenem & $26.6 \pm 3.2^{c}$ & $9.4 \pm 1.2^{c}$ & 2.0 & $\begin{array}{l}N=15 \\
\text { Age } 55.3 \pm 14.3 \text { years } \\
\text { Severe sepsis }\end{array}$ & $\begin{array}{l}\text { Randomized, controlled } \\
\text { cross-over }\end{array}$ & $\begin{array}{l}\text { Thalhammer et al., } 1999 \\
\text { [27] }\end{array}$ \\
\hline Meropenem & $34.4 \pm 15.9$ & $11 \pm 4.3$ & $0.4 \pm 0.12$ & $\begin{array}{l}N=8 \\
\text { Age } 55 \pm 8 \text { years } \\
\text { VAP }\end{array}$ & Descriptive & $\begin{array}{l}\text { de Stoppelaar et al., } \\
2000 \text { [19] }\end{array}$ \\
\hline Meropenem & $19.7 \pm 5$ & $7.3 \pm 3.1$ & $3.1 \pm 1.5$ & $\begin{array}{l}N=14 \\
\text { Age } 73.3 \pm 8.1 \text { years } \\
\text { Severe sepsis }\end{array}$ & Descriptive & $\begin{array}{l}\text { Kitzes-Cohen et al., } \\
2002[21]\end{array}$ \\
\hline Meropenem & $16.0 \pm 3.7^{d}$ & $8.5 \pm 3.2^{d}$ & $1.4 \pm 0.6^{d}$ & $\begin{array}{l}N=9 \\
\text { Age } 39.6 \pm 15.7 \text { years } \\
\text { VAP }\end{array}$ & $\begin{array}{l}\text { Not randomized, } \\
\text { controlled cross-over }\end{array}$ & $\begin{array}{l}\text { Jaruratanasirikul et al., } \\
2005 \text { [20] }\end{array}$ \\
\hline Imipenem & $\begin{array}{l}\text { Imipenem } \\
17.7 \pm 4\end{array}$ & $\begin{array}{l}\text { Imipenem } \\
7.0 \pm 2.5\end{array}$ & $\begin{array}{l}\text { Imipenem } \\
2 \pm 0.3\end{array}$ & $\begin{array}{l}\text { Imipenem } \\
N=10 \\
\text { Age } 65 \pm 19 \text { years }\end{array}$ & $\begin{array}{l}\text { Randomized, parallel } \\
\text { controlled }\end{array}$ & Novelli et al., 2005 [29] \\
\hline Meropenem & $\begin{array}{l}\text { Meropenem } \\
27.1 \pm 7.7\end{array}$ & $\begin{array}{l}\text { Meropenem } \\
11.5 \pm 3.1\end{array}$ & $\begin{array}{l}\text { Meropenem } \\
2.1 \pm 0.5\end{array}$ & $\begin{array}{l}\text { Meropenem } \\
N=10 \\
\text { Age } 67 \pm 19 \text { years } \\
\text { Severe sepsis }\end{array}$ & & \\
\hline Meropenem & $23.8 \pm 4.9$ & $6.7 \pm 4.2$ & $3.7 \pm 1.9$ & $\begin{array}{l}N=6 \\
\text { Age } 65.7 \pm 11.2 \text { years } \\
\text { Peritonitis }\end{array}$ & Descriptive & Karjagin et al., 2008 [25] \\
\hline Meropenem & 22.7 & $13.6 \pm 1.3$ & NR & $\begin{array}{l}N=10 \\
\text { Age range } 48 \text { to } 63 \\
\text { years } \\
\text { Severe sepsis }\end{array}$ & $\begin{array}{l}\text { Randomized, parallel } \\
\text { controlled }\end{array}$ & Roberts et al., 2009 [24] \\
\hline Meropenem & $\begin{array}{l}\text { Meropenem } \\
30.1 \\
{[21.7 \text { to } 53.9]^{\mathrm{e}}}\end{array}$ & $\begin{array}{l}\text { Meropenem } \\
8\left[5 \text { to } 10.99^{e}\right.\end{array}$ & $\begin{array}{l}\text { Meropenem } \\
2.1 \\
{[1.7 \text { to } 3.4]}\end{array}$ & $\begin{array}{l}\text { Meropenem } \\
N=16\end{array}$ & Cross-sectional & Taccone et al., 2010 [23] \\
\hline Piperacillin & $\begin{array}{l}\text { Piperacillin } \\
26.6 \\
{[20.3 \text { to } 30.1]^{\mathrm{e}}}\end{array}$ & $\begin{array}{l}\text { Piperacillin } \\
8.4 \\
{[5.5 \text { to } 18.1]^{\mathrm{e}}}\end{array}$ & $\begin{array}{l}\text { Piperacillin } \\
2.6 \\
{[1.5 \text { to } 3.8]}\end{array}$ & $\begin{array}{l}\text { Piperacillin } \\
N=27\end{array}$ & & \\
\hline Ceftazidime & $\begin{array}{l}\text { Ceftazidime } \\
33.6 \\
{[25.2 \text { to } 49.7]^{\mathrm{e}}}\end{array}$ & $\begin{array}{l}\text { Ceftazidime } \\
3.8 \\
{[2.5 \text { to } 5.5]^{\mathrm{e}}}\end{array}$ & $\begin{array}{l}\text { Ceftazidime } \\
5.8 \\
{[4.1 \text { to } 7.4]}\end{array}$ & $\begin{array}{l}\text { Ceftazidime } \\
N=18\end{array}$ & & \\
\hline Cefepime & $\begin{array}{l}\text { Cefepime } \\
25.2 \\
{[23.1 \text { to } 30.8]^{\mathrm{e}}}\end{array}$ & $\begin{array}{l}\text { Cefepime } \\
5.5 \\
{[4.6 \text { to } 8.4]^{\mathrm{e}}}\end{array}$ & $\begin{array}{l}\text { Cefepime } \\
3.4 \\
{[2.3 \text { to } 5.3]}\end{array}$ & $\begin{array}{l}\text { Cefepime } \\
N=19 \\
\text { All patients: median age } \\
63 \text { years } \\
\text { Severe sepsis or septic } \\
\text { shock }\end{array}$ & & \\
\hline Imipenem & $31.4 \pm 11.7$ & $14.4 \pm 4.5$ & $1.6 \pm 1.3$ & $\begin{array}{l}N=10 \\
\text { Age } 44 \pm 12.2 \text { years } \\
\text { Severe sepsis }\end{array}$ & Descriptive & $\begin{array}{l}\text { McKindley et al., } 1996 \\
\text { [34] }\end{array}$ \\
\hline Imipenem & 18.5 & $6.3 \pm 0.8$ & 2.0 & $\begin{array}{l}N=6 \\
\text { Age } 63.5 \pm 16.7 \text { years } \\
\text { Severe sepsis }\end{array}$ & $\begin{array}{l}\text { Not randomized, } \\
\text { parallel, controlled }\end{array}$ & Tegeder et al., 2002 [32] \\
\hline Imipenem & $45.5 \pm 47.2$ & $12.1 \pm 12.0$ & $2.9 \pm 1.7$ & $\begin{array}{l}N=50 \\
\text { Age } 45.2 \pm 17 \text { years } \\
\text { Presumed Gram- } \\
\text { negative sepsis }\end{array}$ & Cross-sectional & $\begin{array}{l}\text { Belzberg et al., } 2004 \\
\text { [28] }\end{array}$ \\
\hline
\end{tabular}


Table 1 Pharmacokinetic parameters of $\beta$ ? $\beta$ ?-lactam antibiotics ${ }^{\mathrm{a}}$ (Continued)

\begin{tabular}{|c|c|c|c|c|c|c|}
\hline Imipenem & $12.2 \pm 9.9^{f}$ & $12.3 \pm 4.2$ & $N R$ & $\begin{array}{l}N=20 \\
\text { Age } 60.5 \text { years } \\
\text { VAP }\end{array}$ & $\begin{array}{l}\text { Randomized, parallel, } \\
\text { controlled }\end{array}$ & Sakka et al., 2007 [31] \\
\hline Imipenem & $27.2 \pm 6.5$ & $13.3 \pm 5.2$ & $1.4 \pm 0.2$ & $\begin{array}{l}N=6 \\
\text { Age } 53.3 \pm 19.9 \text { years } \\
\text { Severe sepsis }\end{array}$ & $\begin{array}{l}\text { Not randomized, } \\
\text { parallel, controlled }\end{array}$ & Dahyot et al., 2008 [33] \\
\hline Imipenem & $16.7 \pm 5.3^{9}$ & $8.7 \pm 5.3^{9}$ & $1.5 \pm 0.7^{9}$ & $\begin{array}{l}N=9 \\
\text { Age } 63.3 \pm 14.9 \text { years } \\
\text { VAP }\end{array}$ & $\begin{array}{l}\text { Not randomized, } \\
\text { controlled, cross-over }\end{array}$ & $\begin{array}{l}\text { Jaruratanasirikul and } \\
\text { Sudsai, } 2009 \text { [30] }\end{array}$ \\
\hline \multicolumn{7}{|l|}{ Penicillins } \\
\hline Piperacillin & $25.0 \pm 17.2$ & $23.8 \pm 17.2$ & $1.5 \pm 2.1$ & $\begin{array}{l}N=11 \\
\text { Age } 43.6 \pm 15.9 \text { years } \\
\text { Surgical patients }\end{array}$ & Descriptive & $\begin{array}{l}\text { Shikuma et al., } 1990 \\
{[36]}\end{array}$ \\
\hline Piperacillin & $19.5 \pm 3.4^{b}$ & $8.4 \pm 1.4^{b}$ & $1.8 \pm 0.3^{b}$ & $\begin{array}{l}N=10 \\
\text { Age } 37.7 \pm 2.8 \text { years } \\
\text { Burn patients }\end{array}$ & Descriptive & Bourget et al., 1996 [38] \\
\hline Piperacillin & $40.7 \pm 8.7$ & $8.2 \pm 2$ & $4.1 \pm 1.3$ & $\begin{array}{l}N=6 \\
\text { Age } 64 \pm 7 \text { years } \\
\text { Septic shock }\end{array}$ & $\begin{array}{l}\text { Not randomized, } \\
\text { parallel, controlled }\end{array}$ & $\begin{array}{l}\text { Joukhadar et al., } 2001 \\
\text { [44] }\end{array}$ \\
\hline Piperacillin & $34.6 \pm 6.8^{c}$ & $11.8 \pm 4.3^{c}$ & $2.4 \pm 1.2^{c}$ & $\begin{array}{l}N=7 \\
\text { Age range } 45 \text { to } 76 \\
\text { years } \\
\text { Severe sepsis }\end{array}$ & $\begin{array}{l}\text { Not randomized, } \\
\text { controlled, cross-over }\end{array}$ & $\begin{array}{l}\text { Langgartner et al., } 2007 \\
\text { [39] }\end{array}$ \\
\hline Piperacillin & $11.7^{f}$ & 17.2 & 0.4 & $\begin{array}{l}N=13 \\
\text { Age } 37.5 \pm 19.4 \text { years } \\
\text { Severe sepsis }\end{array}$ & $\begin{array}{l}\text { Randomized, parallel, } \\
\text { controlled }\end{array}$ & Roberts et al., 2009 [45] \\
\hline \multicolumn{7}{|l|}{ Cephalosporins } \\
\hline Cefpirome & $23.6 \pm 8.0$ & $8.0 \pm 3.0$ & $2.2 \pm 0.5$ & $\begin{array}{l}N=9 \\
\text { Age } 31 \text { years [19 to } 53 \text { ] } \\
\text { Severe sepsis }\end{array}$ & $\begin{array}{l}\text { Not randomized, } \\
\text { parallel, controlled }\end{array}$ & Jacolot et al., 1999 [47] \\
\hline Cefpirome & $26.4 \pm 7.9$ & $8.8 \pm 3.4$ & $3.1 \pm 1.2$ & $\begin{array}{l}N=12 \\
\text { Age } 41.2 \pm 19 \text { years } \\
\text { Severe sepsis }\end{array}$ & Descriptive & Lipman et al., 2001 [48] \\
\hline Cefpirome & $25.9 \pm 7.1$ & $4.5 \pm 0.7$ & $3.3 \pm 0.5$ & $\begin{array}{l}N=12 \\
\text { Age } 67.2 \pm 8.1 \text { years } \\
\text { Severe sepsis or septic } \\
\text { shock }\end{array}$ & $\begin{array}{l}\text { Not randomized, } \\
\text { parallel, controlled }\end{array}$ & $\begin{array}{l}\text { Joukhadar et al., } 2002 \\
\text { [52] }\end{array}$ \\
\hline Cefpirome & $21.9 \pm 4.5$ & $4.8 \pm 1.6$ & $3.1 \pm 0.9$ & $\begin{array}{l}N=11 \\
\text { Age } 66 \pm 8 \text { years } \\
\text { Severe sepsis }\end{array}$ & $\begin{array}{l}\text { Not randomized, } \\
\text { parallel, controlled }\end{array}$ & $\begin{array}{l}\text { Sauermann et al., } 2005 \\
\text { [51] }\end{array}$ \\
\hline Cefepime & $32.6 \pm 17.5$ & $7.5 \pm 3.1$ & $3.5 \pm 1.1$ & $\begin{array}{l}N=7 \\
\text { Age } 73.7 \pm 4.9 \text { years } \\
\text { Severe sepsis }\end{array}$ & Descriptive & Kieft et al., 1993 [53] \\
\hline Cefepime & $21.8 \pm 5.1$ & $7.6 \pm 2.0$ & $3 \pm 1.2$ & $\begin{array}{l}\mathrm{N}=13 \\
\text { Age } 55 \text { years } \\
\text { Severe sepsis }\end{array}$ & Descriptive & Lipman et al., 1999 [56] \\
\hline Cefepime & $36.1 \pm 11.8$ & $8.8 \pm 2.4$ & $2.8 \pm 0.6$ & $\begin{array}{l}N=12 \\
\text { Age } 41 \pm 13 \text { years } \\
\text { Burn patients }\end{array}$ & Descriptive & $\begin{array}{l}\text { Bonapace et al., } 1999 \\
\text { [57] }\end{array}$ \\
\hline Cefepime & $26.0^{b}$ & $9.1 \pm 1.5^{b}$ & $2.5 \pm 0.6^{b}$ & $\begin{array}{l}N=6 \\
\text { Age } 39.8 \pm 11.3 \text { years } \\
\text { Burn patients }\end{array}$ & Descriptive & Sampol et al., 2000 [61] \\
\hline Cefepime & Cefepime 19.6 & $\begin{array}{l}\text { Cefepime } 7.1 \\
\pm 3.6\end{array}$ & $\begin{array}{l}\text { Cefepime } 2.9 \\
\pm 3.2\end{array}$ & $\begin{array}{l}\text { Cefepime } \\
N=13 \\
\text { Age } 48.2 \pm 21.2 \text { years }\end{array}$ & Cross-sectional & Conil et al., 2007 [54] \\
\hline Ceftazidime & $\begin{array}{l}\text { Ceftazidime } \\
28.8\end{array}$ & $\begin{array}{l}\text { Ceftazidime } \\
7.5 \pm 3.8\end{array}$ & $\begin{array}{l}\text { Ceftazidime } \\
3.1 \pm 2.1\end{array}$ & $\begin{array}{l}\text { Ceftazidime } \\
N=17 \\
\text { Age } 62.9 \pm 22.4 \text { years } \\
\text { Burn patients }\end{array}$ & & \\
\hline Cefepime & $28.7 \pm 13.3^{d}$ & $9.1 \pm 5.6^{d}$ & $4.3 \pm 4.2$ & $\begin{array}{l}N=21 \\
\text { Age } 55.1 \text { years (median) } \\
\text { Nosocomial pneumonia }\end{array}$ & Cross-sectional & Chapuis et al., 2010 [55] \\
\hline
\end{tabular}


Table 1 Pharmacokinetic parameters of $\beta$ ? $\beta$ ?-lactam antibiotics ${ }^{\mathrm{a}}$ (Continued)

\begin{tabular}{|c|c|c|c|c|c|c|}
\hline Ceftazidime & 24.5 & 7.5 & 2.1 & $\begin{array}{l}N=16 \\
\text { Age range } 18 \text { to } 70 \\
\text { years } \\
\text { Pseudomonas infection }\end{array}$ & Descriptive & $\begin{array}{l}\text { Rondanelli et al., } 1986 \\
\text { [64] }\end{array}$ \\
\hline Ceftazidime & $49.3 \pm 18.2^{\mathrm{e}}$ & $15.5 \pm 2.5^{e}$ & $1.8 \pm 0.5^{\mathrm{e}}$ & $\begin{array}{l}N=5 \\
\text { Age } 52.3 \text { years }[21 \text { to } \\
69] \\
\text { VAP }\end{array}$ & $\begin{array}{l}\text { Not randomized, } \\
\text { controlled, cross-over }\end{array}$ & Langer et al., 1991 [76] \\
\hline Ceftazidime & $29.5 \pm 8.7$ & $4.2 \pm 1.9$ & $6.1 \pm 2.5$ & $\begin{array}{l}N=12 \\
\text { Age } 60 \pm 13 \text { years } \\
\text { VAP }\end{array}$ & $\begin{array}{l}\text { Not randomized, } \\
\text { controlled, cross-over }\end{array}$ & $\begin{array}{l}\text { Bressolle et al., } 1992 \\
\text { [77] }\end{array}$ \\
\hline Ceftazidime & $18.9 \pm 9^{c}$ & $5.1^{c}$ & $3.5 \pm 1.6^{c}$ & $\begin{array}{l}N=12 \\
\text { Age } 57 \pm 12 \text { years } \\
\text { Suspected Gram- } \\
\text { negative sepsis }\end{array}$ & $\begin{array}{l}\text { Not randomized, } \\
\text { controlled, cross-over }\end{array}$ & Benko et al., 1996 [67] \\
\hline Ceftazidime & $15.0 \pm 4.3$ & $5.2 \pm 2.2$ & $1.3 \pm 1.2$ & $\begin{array}{l}N=10 \\
\text { Age } 48 \pm 15.1 \text { years } \\
\text { Severe sepsis }\end{array}$ & Descriptive & Young et al., 1997 [65] \\
\hline Ceftazidime & $56.9 \pm 25.9$ & $9.1 \pm 4.8$ & $4.8 \pm 1.9$ & $\begin{array}{l}N=15 \\
\text { Age } 59.3 \pm 14.6 \text { years } \\
\text { Severe sepsis }\end{array}$ & Descriptive & Gómez et al., 1999 [66] \\
\hline Ceftazidime & $\begin{array}{l}22.9[11.8 \text { to } \\
28.1]\end{array}$ & $\begin{array}{l}2.8[0.2 \text { to } \\
7.8]\end{array}$ & $\begin{array}{l}7.7[2 \text { to } \\
44.7]\end{array}$ & $\begin{array}{l}N=21 \\
\text { Age range } 27 \text { to } 73 \\
\text { years } \\
\text { Melioidosis }\end{array}$ & $\begin{array}{l}\text { Not randomized, } \\
\text { parallel, controlled }\end{array}$ & Angus et al., 2000 [71] \\
\hline Ceftazidime & $25.6 \pm 11.2^{c}$ & $11.0 \pm 5.3^{c}$ & $1.7 \pm 0.7^{c}$ & $\begin{array}{l}N=14 \\
\text { Age } 36.1 \pm 12.8 \text { years } \\
\text { Gram-negative } \\
\text { nosocomial pneumonia }\end{array}$ & $\begin{array}{l}\text { Not randomized, } \\
\text { parallel, controlled }\end{array}$ & Hanes et al., 2000 [70] \\
\hline Ceftazidime & $\begin{array}{l}19.6[14 \text { to } \\
28]^{c_{1}} \text { e }\end{array}$ & $\begin{array}{l}5.1[2.3 \text { to } \\
8.9]^{c}\end{array}$ & $\begin{array}{l}4.2[1.3 \text { to } \\
12.3]^{\mathrm{c}}\end{array}$ & $\begin{array}{l}N=6 \\
\text { Age } 64 \text { years [ } 42 \text { to } 87] \\
\text { Surgical peritonitis }\end{array}$ & $\begin{array}{l}\text { Not randomized, } \\
\text { parallel, controlled }\end{array}$ & Buijk et al., 2002 [74] \\
\hline
\end{tabular}

${ }^{a} \mathrm{Cl}$ : clearance; NR: not reported; PK: pharmacokinetics; $\mathrm{T}_{1 / 2}$ : half-life; VAP: ventilator-associated pneumonia; $V_{\mathrm{d}}$ : volume of distribution. ${ }^{\mathrm{b}}$ first-day PK; ${ }^{\mathrm{c} P K}$ after bolus dosing; ${ }^{d}$ PK after 1 -g bolus dosing; ${ }^{\text {f for }} 70 \mathrm{~kg}$; ${ }^{\mathrm{f}}$ central compartment; ${ }^{9} \mathrm{PK}$ after 500 -mg bolus dosing. Except where otherwise indicated, data are means, means \pm standard deviations or medians [interquartile ranges].

119.4 mg $\times$ hour $/ \mathrm{L} ; P=0.001)$, despite a reduction in the dose administered, from $1 \mathrm{~g}$ every $8 \mathrm{~h}$ (tid) to $1 \mathrm{~g}$ every $12 \mathrm{~h}$ (bid) [21].

One study addressed the variability of individual meropenem PK between the first and fourth days of therapy in 11 surgical patients [22]. Despite an increase in $\mathrm{Cr} \mathrm{Cl}$ from a mean of 63.9 to $79.1 \mathrm{~mL} /$ minute during the study period, meropenem $V_{\mathrm{d}}, \mathrm{Cl}$ and AUC remain unchanged. Nevertheless, in another study, by Taccone et al. [23], predefined targets were reached in only $75 \%$ of severe sepsis and septic shock patients after the first dose of $1 \mathrm{~g}$ of meropenem (Table 2), despite the inclusion of patients with acute renal failure $(22 \%)$ who did not receive renal replacement therapy. These authors concluded that PK changes induced by sepsis were largely unpredictable and that none of the evaluated clinical parameters were predictive of PK adequacy: namely, age, severity, presence of shock, use of vasopressors and mechanical ventilation. Also, Roberts et al. [24] showed that the $V_{\mathrm{d}}$ in patients with severe sepsis had great variability, both in the same patient (especially the central compartment: roughly 45\%) and in different patients (nearly 27\%). In their study, despite the fact that all patients had a serum $\mathrm{Cr}<1.36 \mathrm{mg} / \mathrm{dL}$, the meropenem $\mathrm{Cl}$ variability (in the same patient and between patients) still ranged between $10 \%$ and $20 \%$.

The time of infusion of meropenem has also been shown to influence its $\mathrm{T}>\mathrm{MIC}$. In a cross-over study of nine Thai VAP patients [20], after 48 hours of therapy, $1 \mathrm{~g}$ of meropenem tid in 30-minute infusions provided an adequate $\mathrm{T}>\mathrm{MIC}$ in $74.7 \%$ of the patients, for a MIC of $1 \mathrm{mg} / \mathrm{L}$. However, with a MIC of $16 \mathrm{mg} / \mathrm{L}$, only the meropenem regimen of $2 \mathrm{~g}$ tid given in an extended infusion (two hours) led to a $\mathrm{T}>\mathrm{MIC}>40 \%$ [20].

Meropenem tissue PK have been evaluated by microdialysis in several studies (Table 3). The tissue-to-plasma meropenem mean ratio on the first day of antibiotic therapy was found to be 0.74 in the peritoneum [25] and 0.44 in subcutaneous fat [24]. The meropenem CFR was calculated for the 10 patients for whom serum levels were measured in this study according to the Mystic microbiological database [26]. The CFRs were 100\% for Enterobacteriaceae and 40.6\% for Pseudomonas 
Table 2 Pharmacodynamic targets of $\beta$-lactam antibiotics ${ }^{a}$

\begin{tabular}{|c|c|c|c|}
\hline Antibiotics & PD targets & Percentage of patients achieving targets & References \\
\hline \multirow[t]{4}{*}{ Meropenem, $1 \mathrm{~g}$ tid or $3 \mathrm{~g} /$ day $\mathrm{Cl}$} & $\begin{array}{l}40 \% f \mathrm{~T}>\text { MIC, with } f \text { assumed to } \\
\text { be } 98 \% \text {. } \\
\text { CFR according to Mystic database }\end{array}$ & PTA for MIC = 2 mg/L: bolus 100\%, Cl 100\% & $\begin{array}{l}\text { Roberts et al., } \\
2009[24]\end{array}$ \\
\hline & & PTA for MIC = 8 mg/L: bolus 70\%, Cl 100\% & \\
\hline & & CFR for EC: bolus 100\%, Cl 100\% & \\
\hline & & CFR for PA: bolus $40.6 \%$, Cl 100\% & \\
\hline Ceftazidime, $2 \mathrm{~g}$ & $\begin{array}{l}70 \% \mathrm{~T}>4 \times \text { EUCAST breakpoint } \\
\text { of PA }\end{array}$ & $28 \%$ & $\begin{array}{l}\text { Taccone et al., } \\
2010[23]\end{array}$ \\
\hline Cefepime, $2 \mathrm{~g}$ & $\begin{array}{l}70 \% \mathrm{~T}>4 \times \text { EUCAST breakpoint } \\
\text { of PA }\end{array}$ & $16 \%$ & \\
\hline Meropenem, $1 \mathrm{~g}$ & $\begin{array}{l}40 \% \mathrm{~T}>4 \times \text { EUCAST breakpoint } \\
\text { of PA }\end{array}$ & $75 \%$ & \\
\hline Piperacillin/tazobactam, $4.5 \mathrm{~g}$ & $\begin{array}{l}50 \% \mathrm{~T}>4 \times \text { EUCAST breakpoint } \\
\text { of PA }\end{array}$ & $44 \%$ & \\
\hline \multirow[t]{2}{*}{ Imipenem $1 \mathrm{~g}$ tid or $2 \mathrm{~g} /$ day $\mathrm{Cl}$} & $\begin{array}{l}40 \% f \mathrm{~T}>\mathrm{MIC} \text {, with } f \text { assumed to } \\
\text { be } 80 \%\end{array}$ & $\mathrm{MIC}=2 \mathrm{mg} / \mathrm{L}$ bolus dosing 88\%, Cl 100\% & $\begin{array}{l}\text { Sakka et al., } 2007 \\
{[31]}\end{array}$ \\
\hline & & $\mathrm{MIC}=4 \mathrm{mg} / \mathrm{L}$ bolus $75 \%, \mathrm{Cl} 86 \%$ & \\
\hline \multirow[t]{4}{*}{$\begin{array}{l}\text { Piperacillin/tazobactam } 4.5 \mathrm{~g} \text { qid or } 13.5 \\
\mathrm{~g} \mathrm{Cl}\end{array}$} & $\begin{array}{l}50 \% f \mathrm{~T}>\mathrm{MIC} \text {. } \\
\text { CFR according to Mystic database }\end{array}$ & PTA for $\mathrm{MIC}=0.25 \mathrm{mg} / \mathrm{L}$ bolus $79.2 \%, \mathrm{Cl} 100 \%$ & $\begin{array}{l}\text { Roberts et al., } \\
2009[46]\end{array}$ \\
\hline & & PTA for $\mathrm{MIC}=1 \mathrm{mg} / \mathrm{L}$ bolus $60 \%, \mathrm{Cl} 100 \%$ & \\
\hline & & CFR for 18 g/day: bolus 53.4\%, Cl 92.5\% & \\
\hline & & CFR for 13.5 g/day: bolus 40\%, Cl $92.4 \%$ & \\
\hline \multirow[t]{2}{*}{ Cefpirome $2 \mathrm{~g}$ bid } & $60 \% \mathrm{~T}>\mathrm{MIC}$ & $\begin{array}{l}\text { PTA for MIC = } 4 \text { mg/L: bolus 60\%, Cl (4 g/day) } \\
100 \%\end{array}$ & $\begin{array}{l}\text { Lipman et al., } \\
2001[48]\end{array}$ \\
\hline & & $\begin{array}{l}\text { PTA for MIC = } 16 \text { mg/L: bolus 10\%, Cl (4 g/day) } \\
50 \%\end{array}$ & \\
\hline \multirow[t]{2}{*}{ Cefpirome $2 \mathrm{~g}$ tid } & $60 \%$ T > MIC plasma and tissue & PTA for MIC = 4 mg/L: plasma 100\%, tissue 100\% & $\begin{array}{l}\text { Sauermann et al., } \\
2005[51]\end{array}$ \\
\hline & & PTA for MIC = 16 mg/L: plasma 87.5\%, tissue $75 \%$ & \\
\hline \multirow[t]{2}{*}{ Cefpirome $2 \mathrm{~g}$ bid } & $\begin{array}{l}65 \% f \mathrm{~T}>\text { MIC, with } f \text { assumed to } \\
\text { be } 90 \% \text {. } \\
\text { CFR according to EUCAST } \\
\text { database }\end{array}$ & CFR for EC: bolus $99.9 \%, \mathrm{Cl}$ (4 g/day) 100\% & $\begin{array}{l}\text { Roos et al., } 2007 \\
{[50]}\end{array}$ \\
\hline & & CFR for PA: bolus $56.1 \%, \mathrm{Cl}$ (4 g/day) $84.4 \%$ & \\
\hline \multirow[t]{2}{*}{ Cefepime $2 \mathrm{~g}$} & $\begin{array}{l}60 \% \mathrm{~T}>\mathrm{MIC} \\
\mathrm{MIC}=8 \mathrm{mg} / \mathrm{L}(\mathrm{NCCLS} \text { break point } \\
\text { of PA) }\end{array}$ & $\begin{array}{l}\text { PTA with } 1 \mathrm{~g} \text { bid } 45 \% \\
\text { PTA with } 2 \mathrm{~g} \text { bid } 68 \%\end{array}$ & $\begin{array}{l}\text { Bonapace et al., } \\
1999[57]\end{array}$ \\
\hline & & PTA for MIC = 4 mg/L: $1 \mathrm{~g}$ bid 68\%, $2 \mathrm{~g}$ bid 89\% & \\
\hline \multirow[t]{2}{*}{ Cefepime $2 \mathrm{~g}$} & $\begin{array}{l}65 \% f \mathrm{~T}>\text { MIC, with } f \text { assumed to } \\
\text { be } 90 \% \text {. } \\
\text { CFR according to Queensland } \\
\text { Health Pathology Service }\end{array}$ & CFR for EC: $2 \mathrm{~g}$ bid 78.9\%, Cl (4 g/day) 96.9\% & $\begin{array}{l}\text { Roos et al., } 2006 \\
{[60]}\end{array}$ \\
\hline & & CFR for PA: $2 \mathrm{~g}$ bid 54\%, Cl (4 g/day) 91.7\% & \\
\hline Ceftazidime $1 \mathrm{~g}$ every 4 hours & $\begin{array}{l}100 \% \mathrm{~T}>4 \times \mathrm{MIC} \text { (isolated } \\
\text { pathogens; if negative cultures } \\
100 \% \mathrm{~T}>16 \mathrm{mg} / \mathrm{L} \text { ) }\end{array}$ & $\begin{array}{l}\text { Ceftazidime } 47.8 \% \\
\text { PTA with } 1 \mathrm{~g} \text { every } 3 \text { hours } 88.2 \%\end{array}$ & $\begin{array}{l}\text { Conil et al., } 2007 \\
\text { [54] }\end{array}$ \\
\hline Cefepime $2 \mathrm{~g}$ tid & & $\begin{array}{l}\text { Cefepime } 20 \% \\
\text { PTA with } 1 \mathrm{~g} \text { every } 4 \text { hours } 88.2 \%\end{array}$ & \\
\hline \multirow[t]{2}{*}{ Cefepime $2 \mathrm{~g}$ tid } & $\begin{array}{l}50 \% f \mathrm{~T}>\mathrm{MIC} \text {, with } f \text { assumed to } \\
\text { be } 85 \%\end{array}$ & PTA for $\mathrm{MIC}=8 \mathrm{mg} / \mathrm{L} 91.8 \%$ & $\begin{array}{l}\text { Nicasio et al., } \\
2009 \text { [59] }\end{array}$ \\
\hline & & PTA for MIC = $32 \mathrm{mg} / \mathrm{L} 50.3 \%$ & \\
\hline Cefepime $2 \mathrm{~g}$ (each 12 to 36 hours) & $\begin{array}{l}50 \% \mathrm{~T}>\mathrm{MIC} \\
\mathrm{MIC}=8 \mathrm{mg} / \mathrm{L}\end{array}$ & First dose $67 \%$; steady-state $44 \%$ & $\begin{array}{l}\text { Chapuis et al., } \\
2010 \text { [55] }\end{array}$ \\
\hline \multirow[t]{2}{*}{ Ceftazidime $2 \mathrm{~g}$ tid } & $\begin{array}{l}100 \% \mathrm{~T}>5 \times \mathrm{MIC} \\
\mathrm{MIC}=8 \mathrm{mg} / \mathrm{L} \text { (PA break point) }\end{array}$ & $10 \%$ & $\begin{array}{l}\text { Young et al., } 1997 \\
{[65]}\end{array}$ \\
\hline & & PTA for Cl (6 g/day) $60 \%$ & \\
\hline Ceftazidime $2 \mathrm{~g}$ tid or $6 \mathrm{~g} /$ day $\mathrm{Cl}$ & $\begin{array}{l}100 \% \mathrm{~T}>5 \times \mathrm{MIC} \\
\mathrm{MIC}=8 \mathrm{mg} / \mathrm{L} \text { (PA break point) }\end{array}$ & Bolus 20\% & $\begin{array}{l}\text { Lipman et al., } \\
1999[68]\end{array}$ \\
\hline
\end{tabular}




\begin{tabular}{|c|c|c|c|}
\hline & & $\mathrm{Cl} 100 \%$ & \\
\hline \multirow[t]{2}{*}{ Ceftazidime $1.5 \mathrm{~g}$ tid or $4.5 \mathrm{~g} /$ day $\mathrm{Cl}$} & $\begin{array}{l}\text { T }>4 \times \text { MIC plasma and } \\
\text { peritoneum (isolated pathogens) }\end{array}$ & Plasma: bolus dosing 100\%, Cl 100\% & $\begin{array}{l}\text { Buijk et al., } 2002 \\
{[74]}\end{array}$ \\
\hline & & Peritoneum: bolus $88 \%, \mathrm{Cl} 100 \%$ & \\
\hline \multirow[t]{2}{*}{ Ceftazidime 2 to $6 \mathrm{~g} /$ day $\mathrm{Cl}$} & $\begin{array}{l}100 \% \mathrm{~T}>5 \times \mathrm{MIC} \\
\mathrm{MIC}=8 \mathrm{mg} / \mathrm{L} \text { (PA break point) } \\
\text { Target concentration } 40 \pm 10 \mathrm{mg} / \\
\mathrm{L}\end{array}$ & $35.9 \%$ & $\begin{array}{l}\text { Aubert et al., } 2010 \\
\text { [72] }\end{array}$ \\
\hline & & Percentage of time on target (mean) & \\
\hline Meropenem $2 \mathrm{~g}$ tid or $3 \mathrm{~g} \mathrm{Cl}$ & $\begin{array}{l}\text { T > MIC (isolated susceptible } \\
\text { pathogens) }\end{array}$ & Bolus $T=100 \% ; C l T=100 \%$ & $\begin{array}{l}\text { Thalhammer et } \\
\text { al., } 1999[27]\end{array}$ \\
\hline \multirow[t]{2}{*}{ Meropenem $1 \mathrm{~g}$ tid } & T > MIC (isolated pathogens) & $\mathrm{T}=90.8 \%$ & $\begin{array}{l}\text { de Stoppelaar et } \\
\text { al, } 2000[19]\end{array}$ \\
\hline & & $\mathrm{T}>4 \times \mathrm{MIC} \mathrm{T}=52 \%$ & \\
\hline \multirow[t]{2}{*}{ Meropenem $1 \mathrm{~g}$ bid or $1 \mathrm{~g}$ tid } & T > MIC (isolated pathogens) & $\mathrm{T}=80.9 \%(\mathrm{Cr} \mathrm{Cl}>50 \mathrm{~mL} /$ minute; $1 \mathrm{~g}$ tid $)$ & $\begin{array}{l}\text { Kitzes-Cohen et al, } \\
2002[21]\end{array}$ \\
\hline & & $\mathrm{T}=91.7 \%(\mathrm{Cr} \mathrm{Cl}<50 \mathrm{~mL} /$ minute; $1 \mathrm{~g}$ bid $)$ & \\
\hline Imipenem $1 \mathrm{~g}$ tid & $\begin{array}{l}\mathrm{T}>\mathrm{MIC} \text { (isolated sensitive }[\mathrm{MIC} \leq \\
2 \mathrm{mg} / \mathrm{L}] \text { pathogens) }\end{array}$ & $\mathrm{T}=100 \% ; \mathrm{T}>4 \times \mathrm{MIC} \mathrm{T}=87.5 \%$ & $\begin{array}{l}\text { Novelli et al., } 2005 \\
{[29]}\end{array}$ \\
\hline Meropenem $1 \mathrm{~g}$ tid & $\begin{array}{l}\mathrm{T}>\mathrm{MIC} \text { (isolated sensitive }[\mathrm{MIC} \leq \\
2 \mathrm{mg} / \mathrm{L}] \text { pathogens) }\end{array}$ & $\mathrm{T}=100 \% ; \mathrm{T}>4 \times \mathrm{MIC} \mathrm{T}=87.5 \%$ & \\
\hline \multirow[t]{2}{*}{$\begin{array}{l}\text { Meropenem } 1 \mathrm{~g} \text { tid (bolus or 3-hour } \\
\text { infusion) or } 2 \mathrm{~g} \text { tid (3-hour infusion) }\end{array}$} & $\mathrm{T}>\mathrm{MIC}$ & $\begin{array}{l}\text { For } \mathrm{MIC}=1 \mathrm{mg} / \mathrm{L}: 1 \mathrm{~g} \text { tid bolus } \mathrm{T}=74.7 \%, 1 \mathrm{~g} \\
\text { tid } 3 \text { hours } \mathrm{T}=93.6 \%, 2 \mathrm{~g} \text { tid } 3 \text { hours } \mathrm{T}=98.6 \% \mathrm{~s}\end{array}$ & $\begin{array}{l}\text { Jaruratanasirikul et } \\
\text { al., } 2005[20]\end{array}$ \\
\hline & & $\begin{array}{l}\text { For MIC }=16 \mathrm{mg} / \mathrm{L}: 1 \mathrm{~g} \text { tid bolus } \mathrm{T}=28.3 \%, 1 \mathrm{~g} \\
\text { tid } 3 \text { hours } \mathrm{T}=37.8 \%, 2 \mathrm{~g} \text { tid } 3 \text { hours } \mathrm{T}=57.9 \%\end{array}$ & \\
\hline \multirow[t]{2}{*}{ Meropenem $1 \mathrm{~g}$ tid } & $\mathrm{T}>\mathrm{MIC}$ & $\begin{array}{l}\text { For } \mathrm{MIC}=4 \mathrm{mg} / \mathrm{L}: \text { plasma } \mathrm{T}=87 \% \text {, peritoneum } \mathrm{T} \\
=87 \%\end{array}$ & $\begin{array}{l}\text { Karjagin et al., } \\
2008[25]\end{array}$ \\
\hline & & $\begin{array}{l}\text { For } \mathrm{MIC}=16 \mathrm{mg} / \mathrm{L}: \text { plasma } \mathrm{T}=55 \% \text {, peritoneum } \\
\mathrm{T}=43 \%\end{array}$ & \\
\hline \multirow[t]{2}{*}{$\begin{array}{l}\text { Imipenem } 500 \mathrm{mg} \text { qid ( } 30 \text { minutes or } \\
\text { 2-hour infusion) or } 1 \mathrm{~g} \text { qid (2-hour } \\
\text { infusion) }\end{array}$} & $\mathrm{T}>\mathrm{MIC}$ & $\begin{array}{l}\text { For } \mathrm{MIC}=1 \mathrm{mg} / \mathrm{L}: 500 \mathrm{mg} \text { qid } 30 \text { minutes } \mathrm{T}= \\
64.7 \%, 500 \mathrm{mg} \text { qid } 2 \text { hours } \mathrm{T}=76.5 \%, 1 \mathrm{~g} \text { qid } 2 \\
\text { hours } \mathrm{T}=93.4 \%\end{array}$ & $\begin{array}{l}\text { Jaruratanasirikul } \\
\text { and Sudsai, } 2009 \\
\text { [30] }\end{array}$ \\
\hline & & $\begin{array}{l}\text { For } \mathrm{MIC}=4 \mathrm{mg} / \mathrm{L}: 500 \mathrm{mg} \text { qid } 30 \text { minutes } \mathrm{T}= \\
20.3 \%, 500 \mathrm{mg} \text { qid } 2 \text { hours } \mathrm{T}=17.7 \%, 1 \mathrm{~g} \text { qid } 2 \\
\text { hours } \mathrm{T}=60.3 \%\end{array}$ & \\
\hline \multirow[t]{2}{*}{ Piperacillin $3 \mathrm{~g}$ qid or $8 \mathrm{~g} /$ day $\mathrm{Cl}$} & $\mathrm{T}>\mathrm{MIC}$ & $\begin{array}{l}\text { For MIC = } 16 \mathrm{mg} / \mathrm{L}: \text { bolus dosing } \mathrm{T}=62 \%, \mathrm{Cl} \mathrm{T}= \\
100 \%\end{array}$ & $\begin{array}{l}\text { Rafati et al., } 2006 \\
\text { [40] }\end{array}$ \\
\hline & & For MIC $=32 \mathrm{mg} / \mathrm{L}:$ bolus $\mathrm{T}=39 \%, \mathrm{Cl} \mathrm{T}=65 \%$ & \\
\hline Cefepime $2 \mathrm{~g}$ bid & $\begin{array}{l}\mathrm{T}>\mathrm{MIC} \\
\mathrm{MIC}=7 \mathrm{mg} / \mathrm{L}\left(\mathrm{MIC}_{90} \text { of PA) }\right.\end{array}$ & $\mathrm{T}=80 \%$ & $\begin{array}{l}\text { Kieft et al., } 1993 \\
\text { [53] }\end{array}$ \\
\hline Ceftazidime $2 \mathrm{~g}$ tid or $3 \mathrm{~g} /$ day $\mathrm{Cl}$ & $\begin{array}{l}\mathrm{T}>\mathrm{MIC} \\
\mathrm{MIC}=4 \mathrm{mg} / \mathrm{L} \text { (MIC of one isolated } \\
\mathrm{PA})\end{array}$ & Bolus T $=92 \% ; \mathrm{Cl} T=100 \%$ & $\begin{array}{l}\text { Benko et al., } 1996 \\
{[67]}\end{array}$ \\
\hline Ceftazidime $2 \mathrm{~g}$ tid or $60 \mathrm{mg} / \mathrm{kg} /$ day $\mathrm{Cl}$ & T > MIC (isolated pathogens) & Bolus $\mathrm{T}=92.9 \% ; \mathrm{Cl} \mathrm{T}=100 \%$ & $\begin{array}{l}\text { Hanes et al., } 2000 \\
\text { [70] }\end{array}$ \\
\hline
\end{tabular}

$\overline{\mathrm{a} A B}$ : Acinetobacter baumanii; bid: dose every 12 hours; CFR: cumulative fraction of response; Cl: continuous infusion; Cr Cl: creatinine clearance; EC: Escherichia coli; EUCAST: European Committee on Antimicrobial Susceptibility Testing; $f$ : free drug fraction; KP: Klebsiella pneumoniae; MIC: minimal inhibitory concentration; MIC 90 : $90^{\text {th }}$ percentileof MIC in a bacteria population; NCCLS: National Committee for Clinical Laboratory Standards; PA: Pseudomonas aeruginosa; PD: pharmacodynamics; PTA: probability of target attainment; qid: dose every 6 hours; SA: Staphylococcus aureus; T > MIC: time that antibiotic concentration is above bacteria MIC; tid: dose every 8 hours.

aeruginosa after bolus dosing, whilst with continuous infusion they were $100 \%$ for both bacteria, despite the use of a small daily dose ( $2 \mathrm{~g} /$ day $)$ [27].

\section{Imipenem}

In ICU patients, increased $V_{\mathrm{d}}$ and $\mathrm{Cl}$ of imipenem have also been reported (Table 1). Therefore, its $T_{1 / 2}$ and $T>$ MIC may be difficult to predict, depending on the relative changes of these two parameters. This difficulty was shown by Belzberg et al. [28] in a cohort of ICU surgical and trauma patients with presumed Gram-negative sepsis. In this relatively young population (mean age $45.2 \pm$ 17 years and mean body weight $79.7 \pm 17.7 \mathrm{~kg}$ ), $44 \%$ of patients presented trough levels lower than the intended $4 \mathrm{mg} / \mathrm{L}$ at steady state. A mean $\mathrm{Cr} \mathrm{Cl}$ of $103.8 \mathrm{~mL} / \mathrm{min}$ ute was found, but with large variability: two patients had renal failure and nineteen patients had a $\mathrm{Cr} \mathrm{Cl}>$ 
Table 3 Tissue penetration of $\beta$-lactams ${ }^{a}$

\begin{tabular}{|c|c|c|c|c|}
\hline Antibiotics & Samples & Patient demographics & Concentration ratios $^{\mathrm{b}}$ & References \\
\hline \multicolumn{5}{|c|}{ Muscle and subcutaneous tissue } \\
\hline Meropenem & $\begin{array}{l}\text { Microdialysis in subcutaneous } \\
\text { tissue }\end{array}$ & $\begin{array}{l}N=10 \text { severe sepsis, } 5 \\
\text { continuous infusion }\end{array}$ & $\begin{array}{l}\text { Bolus } 0.44 \\
\text { Continuous infusion } 0.57 \text { (day 2) }\end{array}$ & $\begin{array}{l}\text { Roberts et al., } 2009 \\
{[24]}\end{array}$ \\
\hline \multirow[t]{6}{*}{ Imipenem } & $\begin{array}{l}\text { Microdialysis in muscle and } \\
\text { subcutaneous tissue }\end{array}$ & $\begin{array}{l}N=11 \text { ( } 6 \text { patients) } \\
\text { Severe sepsis }\end{array}$ & Patients & $\begin{array}{l}\text { Tegeder et al., } 2002 \\
\text { [32] }\end{array}$ \\
\hline & & & - Muscle 0.1 & \\
\hline & & & - Subcutaneous 0.14 & \\
\hline & & & Volunteers & \\
\hline & & & • Muscle 0.5 & \\
\hline & & & - Subcutaneous 0.43 & \\
\hline Imipenem & Microdialysis in muscle & $\begin{array}{l}N=12 \text { (6 patients) } \\
\text { Severe sepsis }\end{array}$ & $\begin{array}{l}\text { Patients } 1 \\
\text { Volunteers } 0.97\end{array}$ & $\begin{array}{l}\text { Dahyot et al., } 2008 \\
\text { [33] }\end{array}$ \\
\hline \multirow[t]{6}{*}{ Piperacillin } & $\begin{array}{l}\text { Microdialysis in muscle and } \\
\text { subcutaneous tissue }\end{array}$ & $\begin{array}{l}N=12 \text { (6 patients) } \\
\text { Septic shock }\end{array}$ & Patients & $\begin{array}{l}\text { Joukhadar et al., } \\
2001[44]\end{array}$ \\
\hline & & & - Muscle 0.19 & \\
\hline & & & - Subcutaneous 0.1 & \\
\hline & & & Volunteers & \\
\hline & & & - Muscle 0.55 & \\
\hline & & & - Subcutaneous 0.31 & \\
\hline Piperacillin & $\begin{array}{l}\text { Microdialysis in subcutaneous } \\
\text { tissue }\end{array}$ & $\begin{array}{l}N=13 \\
\text { Severe sepsis }\end{array}$ & $\begin{array}{l}\text { Bolus } 0.21 \\
\text { Continuous infusion } 0.2\end{array}$ & $\begin{array}{l}\text { Roberts et al., } 2009 \\
\text { [45] }\end{array}$ \\
\hline Cefpirome & Microdialysis in muscle & $\begin{array}{l}N=18 \text { ( } 12 \text { patients) } \\
\text { Severe sepsis or septic shock }\end{array}$ & $\begin{array}{l}\text { Patients } 0.63 \\
\text { Volunteers } 0.83\end{array}$ & $\begin{array}{l}\text { Joukhadar et al., } \\
2002[52]\end{array}$ \\
\hline Cefpirome & $\begin{array}{l}\text { Microdialysis in subcutaneous } \\
\text { tissue }\end{array}$ & $\begin{array}{l}N=18 \text { (11 patients) } \\
\text { Severe sepsis }\end{array}$ & $\begin{array}{l}\text { Patients } 0.43 \\
\text { Volunteers } 0.79\end{array}$ & $\begin{array}{l}\text { Sauermann et al., } \\
2005[51]\end{array}$ \\
\hline \multicolumn{5}{|l|}{ Burned skin } \\
\hline Cefepime & Biopsy of burned area & $\begin{array}{l}N=6 \\
\text { Burn patients }\end{array}$ & $\begin{array}{l}\text { Day } 31.52 \text { (point concentration } 3 \text { to } 5 \\
\text { hours after dose) }\end{array}$ & $\begin{array}{l}\text { Sampol et al., } 2000 \\
{[61]}\end{array}$ \\
\hline \multicolumn{5}{|l|}{ Peritoneum } \\
\hline Meropenem & Microdialysis in peritoneum & $\begin{array}{l}N=6 \\
\text { Surgical peritonitis }\end{array}$ & 0.74 & $\begin{array}{l}\text { Karjagin et al., } 2008 \\
{[25]}\end{array}$ \\
\hline \multirow[t]{2}{*}{ Ceftazidime } & Peritoneal drainage & $\begin{array}{l}N=18 \\
\text { Surgical peritonitis }\end{array}$ & $\begin{array}{l}\text { Day } 2 \\
\cdot \text { Continuous infusion } 0.56\end{array}$ & Buijk et al., 2002 [74] \\
\hline & & & • Bolus 0.35 & \\
\hline Imipenem & ELF (bronchoscopy) & $\begin{array}{l}N=8 \\
\text { Pneumonia }\end{array}$ & $\begin{array}{l}0.20 \text { (point concentration ratio } 2 \text { hours } \\
\text { after dose) }\end{array}$ & $\begin{array}{l}\text { Muller-Serieys et al., } \\
1987 \text { [35] }\end{array}$ \\
\hline Imipenem & $\begin{array}{l}\text { Bronchial secretions (tracheal } \\
\text { aspirate) }\end{array}$ & $\begin{array}{l}N=10 \\
\text { Trauma patients with VAP }\end{array}$ & NR & $\begin{array}{l}\text { McKindley et al., } \\
1996[34]\end{array}$ \\
\hline Piperacillin & ELF (bronchoscopy) & $\begin{array}{l}N=10 \\
\operatorname{VAP}\end{array}$ & $\begin{array}{l}0.57 \text { (point concentration ratio } 5 \text { hours } \\
\text { after dose) }\end{array}$ & $\begin{array}{l}\text { Boselli et al., } 2004 \\
\text { [41] }\end{array}$ \\
\hline Piperacillin & ELF (bronchoscopy) & $\begin{array}{l}N=40 \\
\operatorname{VAP}\end{array}$ & $\begin{array}{l}0.44 \text { (point concentration ratio } 4 \text { hours } \\
\text { after dose) }\end{array}$ & $\begin{array}{l}\text { Boselli et al., } 2008 \\
\text { [43] }\end{array}$ \\
\hline Piperacillin & $\begin{array}{l}\text { Bronchial secretions (tracheal } \\
\text { aspirate) }\end{array}$ & $\begin{array}{l}N=8 \\
\text { VAP }\end{array}$ & 0.36 & Jehl et al., 1994 [42] \\
\hline Cefepime & ELF (bronchoscopy) & $\begin{array}{l}N=20 \\
\operatorname{VAP}\end{array}$ & 1.04 (point concentration ratio) & $\begin{array}{l}\text { Boselli et al., } 2003 \\
\text { [63] }\end{array}$ \\
\hline \multirow[t]{2}{*}{$\begin{array}{l}\text { Cefepime or } \\
\text { ceftazidime }\end{array}$} & $\begin{array}{l}\text { Bronchial secretions (tracheal } \\
\text { aspirate) }\end{array}$ & $\begin{array}{l}N=5 \text { cefepime } \\
\text { VAP }\end{array}$ & Cefepime $<0.02$ & $\begin{array}{l}\text { Klekner et al., } 2006 \\
\text { [62] }\end{array}$ \\
\hline & & $\begin{array}{l}N=4 \text { ceftazidime } \\
\text { VAP }\end{array}$ & Ceftazidime $<0.05$ & \\
\hline Ceftazidime & $\begin{array}{l}\text { Bronchial secretions (tracheal } \\
\text { aspirate) }\end{array}$ & $\begin{array}{l}N=5 \\
\text { Pneumonia }\end{array}$ & 0.12 & $\begin{array}{l}\text { Langer et al., } 1991 \\
{[76]}\end{array}$ \\
\hline Ceftazidime & $\begin{array}{l}\text { Bronchial secretions (tracheal } \\
\text { aspirate) }\end{array}$ & $\begin{array}{l}N=12 \\
\text { Nosocomial pneumonia }\end{array}$ & 0.76 & $\begin{array}{l}\text { Bressolle et al., } 1992 \\
\text { [77] }\end{array}$ \\
\hline Ceftazidime & ELF (bronchoscopy) & $\begin{array}{l}N=15 \\
\operatorname{VAP}\end{array}$ & $\begin{array}{l}0.21 \text { (point concentration ratio at } \\
\text { steady state) }\end{array}$ & $\begin{array}{l}\text { Boselli et al., } 2004 \\
\text { [69] }\end{array}$ \\
\hline
\end{tabular}

${ }^{a}$ ELF: epithelial lining fluid; NR: not reported. ${ }^{b}$ Mean area under the concentration time curve (AUC) tissue-to-plasma ratio unless otherwise stated. 
$120 \mathrm{~mL} /$ minute. Nevertheless, no correlation was found between PK parameters and body weight, severity of disease, blood pressure or renal function [28].

Another study compared meropenem and imipenem first-dose $\mathrm{PK}$ in patients with normal renal function (serum $\mathrm{Cr}<1.5 \mathrm{mg} / \mathrm{dL}$ ). Again, both $V_{\mathrm{d}}$ and $\mathrm{Cl}$ were significantly elevated, although more so in the meropenem group [29]. However, their T > MIC for sensitive isolated pathogens were similar. Again, there was a relationship between $\mathrm{Cr} \mathrm{Cl}$ and $\mathrm{T}_{1 / 2}$ : Patients with a $\mathrm{Cr} \mathrm{Cl}$ $<50 \mathrm{~mL} /$ minute had a significantly longer $\mathrm{T}_{1 / 2}$ for both antibiotics.

The PD efficacy of imipenem is also influenced by the dose and the time of infusion [30]. Using PK data from a cross-over steady-state study of VAP patients, Jaruratanasirikul and Sudsai [30] showed by modelling of imipenem PD that, for a MIC of $4 \mathrm{mg} / \mathrm{L}$, a 500-mg dose delivered every 6 hours (qid) for 30 minutes achieved a T > MIC of $64.7 \%$ and increased to $76.5 \%$ with a 2 -hour infusion. However, this study excluded shock and renal failure patients ( $\mathrm{Cr} \mathrm{Cl}<60 \mathrm{~mL} /$ minute). With PD modelling of PK data derived from another 20 VAP patients [31], continuous infusion led to improved PTA despite the use of lower dosages (Table 2). In this latter study, all patients had $f$ imipenem $\mathrm{T}>\mathrm{MIC}$ of $100 \%$, but three patients died.

Tissue microdialysis had been used to assess imipenem PK, but with very dissimilar results (Table 3 ): namely, the tissue-to-plasma ratio. This has been found to be markedly depressed in a cohort of severe critically ill patients compared to healthy volunteers (subcutaneous tissue-to-plasma 0.14 vs. 0.43 and muscle tissueto-plasma 0.11 vs. 0.5, respectively) [32]. However, Dahyot et al. [33] disputed these results and found $f$ imipenem in plasma and muscle to be virtually superimposed at any time, both in patients and in healthy volunteers. Some differences exist between these two studies. In the Tegeder et al. study [32], the patients had lower $\mathrm{Cr} \mathrm{Cl}$ (medians $32.8 \mathrm{~mL} /$ minute vs. $156 \mathrm{~mL}$ / minute) and samples were collected at steady state and not after the first dose. Moreover, Dahyot et al. [33] accounted only for the $f$ imipenem in plasma and found higher imipenem $V_{\mathrm{d}}$ and $\mathrm{Cl}$. Different methods of calculating in vivo microdialysis recovery rates may also explain some of the diverse observed results. Nevertheless, low imipenem penetration ratios, as low as 0.06 $[34,35]$, in bronchial secretions were reported in pneumonia patients (Table 3).

\section{Penicillins}

\section{Piperacillin}

Similarly to other $\beta$-lactams, piperacillin $V_{\mathrm{d}}$ and $\mathrm{Cl}$ have generally been found to be increased in ICU patients (Table 1). However, most studies have excluded renal failure patients.
Piperacillin $\mathrm{Cl}$ and trough concentrations were strongly related to $\mathrm{Cr} \mathrm{Cl}$ [36-38]. Taccone et al. [23] showed that only $15 \%$ of patients with high $\mathrm{Cr} \mathrm{Cl}(>50$ $\mathrm{mL} /$ minute) maintained piperacillin concentrations $>$ $50 \%$ of $\mathrm{T}>4 \times \mathrm{MIC}$ after the first antibiotic dose, as opposed to $71 \%$ of patients with lower $\mathrm{Cr} \mathrm{Cl}(P=0.03)$. In contrast, in 10 young burn patients (mean total burned area $40.8 \pm 3.1 \%$ ) with a mean $\mathrm{Cr} \mathrm{Cl}$ of 119.8 $\mathrm{mL} / \mathrm{minute}$ and Pseudomonas aeruginosa infection, the authors found a $20 \%$ increase in $\mathrm{T}_{1 / 2}$ after the first dose of antibiotic compared to the third day of therapy, which was related to a larger $V_{\mathrm{d}}$ (mean of $19.6 \mathrm{~L}$ vs. 16.4 L) [38]. Overall, the piperacillin AUC was similar in the two measurements (mean of $640 \mathrm{mg} \times$ hour/L vs. $622 \mathrm{mg} \times$ hour/L).

Piperacillin is stable for at least 24 hours at room temperature, making it a suitable choice for continuous infusion. With this strategy, higher steady-state concentrations are expected, theoretically providing a higher $\mathrm{T}$ $>$ MIC even with the use of a lower daily dose [39]. A study by Rafati et al. [40] also supports this strategy. These authors showed that, for a MIC $=16 \mathrm{mg} / \mathrm{L}$, the $\mathrm{T}$ $>$ MIC was higher with continuous infusions ( $8 \mathrm{~g} /$ day) than with bolus dosing ( $3 \mathrm{~g}$ tid) (100\% vs. 62\%, respectively). However, the mortality rate was similar.

In VAP patients, piperacillin showed good penetration in bronchial secretions [41-43]. Nevertheless, its epithelial lining fluid (ELF) steady-state concentration was lower than the MIC for Pseudomonas aeruginosa after a 4.5-g tid dose [41]. With continuous infusion, an increase in pulmonary concentration was found, at least in the subset of patients with moderate renal failure (measured $\mathrm{Cr} \mathrm{Cl}<50 \mathrm{~mL} /$ minute), about three times higher than in the patients with normal renal function [43]. However, no relationship was found between ELF piperacillin concentration and clinical success. Similar concentrations were found in the eight patients who died or had persistent infections and in those who experienced therapeutic success [43].

Subcutaneous tissue-to-plasma ratio and PK have been assessed in microdialysis studies. In six septic shock patients (mean norepinephrine dose $0.8 \mu \mathrm{g} / \mathrm{kg} /$ minute) [44], the subcutaneous tissue-to-plasma AUC ratio was only 0.1 , one-third of that measured in healthy volunteers. Peak tissue concentration was also delayed in patients (122 minutes in patients compared with 27 minutes in healthy volunteers), and $T_{1 / 2}$ in tissues was nearly nine times longer. In 13 younger patients with less severe sepsis [45], the AUC tissue-to-plasma ratio was roughly 0.2 . In accordance with their serum PK (as well as PK of another five patients) [46], piperacillin/ tazobactam CFR was calculated to be $92.3 \%$ with continuous infusion (13.5 g/day) and $53.4 \%$ with bolus dosing (4.5 g qid, or $18 \mathrm{~g} /$ day). Again, no correlation was found 
between tissue concentration and outcomes. Despite the low tissue concentration levels, all patients in both groups survived [45].

\section{Cephalosporins Cefpirome}

Cefpirome PK studies have produced heterogeneous results. A 2-g dose was adequate in young trauma patients $(\mathrm{Cr} \mathrm{Cl} \geq 50 \mathrm{~mL} / \mathrm{minute})$ and in similar healthy volunteers. After the first dose, the mean $\mathrm{T}>$ MIC were $75 \%$ and $80 \%$, respectively (with a MIC of 4 $\mathrm{mg} / \mathrm{L}, P=0.76$ ) [47]. However, in 12 similar patients, a lower $\mathrm{T}>\mathrm{MIC}(60 \%)$ was found, which was probably related to higher cefpirome $\mathrm{Cl}$ [48]. After four days of therapy, the cefpirome mean PK parameters remained similar ( $\mathrm{T}>\mathrm{MIC} 67 \%$ and AUC $242 \mathrm{mg} \times$ hour/L vs. $306 \mathrm{mg} \times$ hour/L at steady state). Further analyses [49] showed a strong correlation between $\mathrm{Cr}$ $\mathrm{Cl}$ and either cefpirome or cefepime $\mathrm{Cl}\left(r^{2}=0.81\right)$. Patients with the lower range of $\mathrm{T}>\mathrm{MIC}$ had a higher $\mathrm{Cr} \mathrm{Cl}$, usually above $144 \mathrm{~mL} / \mathrm{minute}$ [49]. According to these measured PK data, the authors performed a simulation to demonstrate improved CFR of cefpirome given as a continuous infusion to treat Pseudomonas aeruginosa infection, from $56.1 \%$ to 84.4\% (Table 2) [50].

Cefpirome tissue PK were evaluated on the basis of microdialysis. Sauermann et al. [51] found a low subcutaneous tissue concentration in patients with severe sepsis, almost half of healthy volunteers, despite a longer plasma $\mathrm{T}_{1 / 2}(183$ minutes vs. 95 minutes; $P<0.05)$. Similar results were reported by Joukhadar et al. [52], who found muscle-to-plasma ratios of 0.63 in patients and 0.83 in healthy volunteers (Table 3 ).

\section{Cefepime}

Roughly a twofold variation of cefepime $V_{\mathrm{d}}$ has been reported in PK studies (Table 1 ) of severe sepsis and septic shock patients [23], elderly septic patients [53], young burn patients [54] and nosocomial pneumonia patients [55]. Cefepime $\mathrm{Cl}$ has also been found to be closely correlated with $\mathrm{Cr} C l$ in this last listed cohort $\left(r^{2}\right.$ $=0.77)$ [55], in another cohort of septic patients $\left(r^{2}=\right.$ 0.74) [56] and in burn patients $\left(r^{2}=0.58\right)$ [57]. Therefore, patients with renal dysfunction may experience toxicity.

In 21 septic patients receiving cefepime at a dose of 2 $\mathrm{g}$ bid, more than twofold peak variations and roughly 40-fold trough variations were observed. Again, the cefepime $\mathrm{Cl}$ correlated with $\mathrm{Cr} \mathrm{Cl}\left(r^{2}=0.77\right)$. Two patients with low $\mathrm{Cr} \mathrm{Cl}$ (19 and $12 \mathrm{~mL} /$ minute) had trough levels > $20 \mathrm{mg} / \mathrm{L}$ despite dosage adjustment. They both had neurologic symptoms (namely, confusion and muscle jerks) that were not identified as toxicity but resolved promptly after drug arrest [55].
A cefepime bolus of $2 \mathrm{~g}$ bid was found to be insufficient to reach a high PD target after the first dose (Table 2), both in $80 \%$ of young burn patients (burn area $21.8 \%)$ with high mean $\mathrm{Cr} \mathrm{Cl}(119.2 \mathrm{~mL} /$ minute) [54] and in the Taccone et al. study [23], in which only $16 \%$ of patients achieved the intended target.

Two other studies have evaluated cefepime PK, one of which addressed the first day of therapy for 55 nosocomial pneumonia or bacteraemia patients (67\% trauma) [58] and the other of which described the status of 32 VAP patients on the second day of cefepime treatment [59]. Both studies unveiled a relationship between $V_{\mathrm{d}}$ and total body weight as well as between excretion, either elimination rate constant [59] or $\mathrm{Cl}$ [58], and $\mathrm{Cr}$ $\mathrm{Cl}$. However, significant interpatient variability was again observed, with regard to both cefepime $\mathrm{Cl}$ (58\%) and central compartment $V_{\mathrm{d}}(67 \%)$ [58].

A PD model was developed with this VAP population PK data: despite a 2-g tid dose, PTA > 90\% was achieved only with a MIC $\leq 8 \mathrm{mg} / \mathrm{L}$ [59]. In another cefepime PD model, the CFR of a 2-g bid dose, used to treat both Escherichia coli and Klebsiella pneumoniae, was 78.9\%. However, for Pseudomonas aeruginosa, CFR was only $53.6 \%$ (Table 2) and increased with either $2 \mathrm{~g}$ tid or continuous infusion ( $4 \mathrm{~g} /$ day or $6 \mathrm{~g} /$ day) to $84.9 \%$, 91.7\% and $94.8 \%$ respectively. Nevertheless, the CFR for Acinetobacter baumanii [60], even with a continuous infusion of $6 \mathrm{~g} /$ day, was only 75\%, reemphasizing the importance of appropriate dosing and the potential benefit of continuous infusion against difficult-to-treat bacteria.

Also, the cefepime tissue concentration was assessed in biopsy samples collected from the skin of burn patients three to five hours after a bolus dose on day 3 of antibiotic therapy. A mean biopsy-to-plasma cefepime ratio of 1.5 (range 0.4 to 5.1) was found [61]. Klekner et al. [62] were unable to detect cefepime in bronchial secretions from any of the five studied patients six hours after an $80 \mathrm{mg} / \mathrm{kg}$ dose. However, using continuous infusion ( $4 \mathrm{~g} /$ day) to treat VAP patients, Boselli et al. [63] found, at steady state, higher and similar plasma and ELF concentrations (mean of $13.5 \mathrm{mg} / \mathrm{L}$ and 14.1 $\mathrm{mg} / \mathrm{L}$, respectively). Although different sampling methods may have influenced these differences, continuous infusion seems to prolong $\mathrm{T}>\mathrm{MIC}$ in the lungs. Nevertheless, no correlation with therapeutic outcomes was reported.

\section{Ceftazidime}

Several studies have shown ceftazidime PK heterogeneity in ICU septic patients with Pseudomonas infections (mostly nosocomial pneumonia) [64], severe sepsis $[65,66]$ and burns [54]. Similarly to other $\beta$-lactams, the authors noted a large variation of both $V_{\mathrm{d}}$ and $\mathrm{Cl}$ (Table 1 ) and consequently significant interpatient variability in 
$\mathrm{T}_{1 / 2}$ and trough concentrations. Also, a correlation between $\mathrm{Cl}$ and $\mathrm{Cr} \mathrm{Cl}$ was usually reported $[65,66]$.

Continuous infusion of ceftazidime was compared with bolus dosing in five different studies [67-71]. In all, there was an increase in $\mathrm{T}>\mathrm{MIC}$ with continuous infusion despite lower daily doses. However, only in severe melioidosis was this strategy associated with lower mortality (3 of 10 patients vs. 9 of 11 patients) [71]. Those patients had low $\mathrm{Cr} \mathrm{Cl}(26.6 \mathrm{~mL} /$ minute) and received ceftazidime dosages adjusted to their body weight (4 $\mathrm{mg} / \mathrm{kg} /$ hour or $40 \mathrm{mg} / \mathrm{kg}$ tid, for a mean body weight of $49.4 \mathrm{~kg}$ ). Ceftazidime steady-state concentration was measured in another cohort of 92 patients receiving continuous infusions [72]. Therapeutic drug monitoring was performed on the second day of therapy. The mean serum concentration was $46.9 \mathrm{mg} / \mathrm{L}$, but again with a very wide range of serum concentrations (7.4 to 162.3 $\mathrm{mg} / \mathrm{L}$ ). Therefore, dosage modification was common because of low serum levels (36.9\%) and high serum levels $(27.2 \%)$, with the latter being associated with lower $\mathrm{Cr} \mathrm{Cl}$ (mean of $51 \mathrm{~mL} /$ minute compared with 103 $\mathrm{mL} /$ minute for patients with low serum levels). Similar results were shown in another large ceftazidime PK study assessing a mixed septic population with a higher mean $\mathrm{Cr} \mathrm{Cl}$ (123 mL/minute) [73]. The lower $\mathrm{T}>\mathrm{MIC}$ was found in patients with the higher $\mathrm{Cr} \mathrm{Cl}$, especially after bolus dosing (Table 2).

Continuous infusion of ceftazidime $(4.5 \mathrm{~g} /$ day $)$ was also associated with a higher peritoneal AUC at day 2 compared to bolus dosing $(1.5 \mathrm{~g}$ tid $)$ in surgical patients with peritonitis $(522 \mathrm{mg} \times$ hour/L vs. $316 \mathrm{mg} \times$ hour $/ \mathrm{L}$; $P=0.01$ ) [74], despite similar serum AUC (and $\mathrm{Cr} \mathrm{Cl}>$ $30 \mathrm{~mL} /$ minute). Therefore, although serum $\mathrm{T}>4 \times$ MIC was $>90 \%$ in all patients, peritoneal $\mathrm{T}>4 \times$ MIC was still $>90 \%$ with continuous infusion but only $44 \%$ with bolus dosing. Nevertheless, no difference in mortality was noted ( $25 \%$ vs. $33 \% ; P=1.0)$. A PD model of ceftazidime in ICU patients also showed higher PTA with continuous infusion ( $100 \%$ for MIC $\leq 8 \mathrm{mg} / \mathrm{L}$ ) than with bolus dosing [75].

Ceftazidime concentration in bronchial secretions was measured in four studies of VAP patients. Very low concentrations, $<0.5 \mathrm{mg} / \mathrm{L}$ and $<0.3 \mathrm{mg} / \mathrm{L}$, were found in two of them [62,76]. Bressole et al. [77] found a higher ratio between bronchial secretions and plasma concentration (0.76) in patients infected after abdominal surgery. A longer $\mathrm{T}_{1 / 2}(6.1$ hours $)$ and a lower $\mathrm{Cl}(4.2 \mathrm{~L} /$ hour) may explain some of these differences. With continuous infusion, a ratio of 0.21 between ELF and serum was observed [69].

\section{Discussion}

In our systematic review, we have aggregated information from 57 prospective studies related to the PK of $\beta$ - lactam antibiotics, which are among the most often agents used to treat sepsis in ICU patients [78]. Overall, an increased $V_{\mathrm{d}}$ of all the studied antibiotics was reported (Figure 3), which was related to total body weight $[58,73]$, but with significant variability. Drug $\mathrm{Cl}$ was also increased and usually related to $\mathrm{Cr} \mathrm{Cl}$. Those changes were largely unpredictable, with important interpatient variability. However, the higher $\mathrm{Cl}$ values were noted in studies that excluded patients with renal dysfunction, a common strategy, which may limit the interpretation of the data reported.

Therapeutic drug monitoring was rarely performed. In addition, data on the daily variation of PK parameters in ICU patients, as well as the ideal frequency of this monitoring, are currently limited. Nevertheless, two of the reviewed studies $[55,72]$ showed that inadequate dosing may be common in this population and may jeopardize $\beta$-lactam antibiotics efficacy or even lead to toxicity [79]. Roberts et al. [80] measured piperacillin/tazobactam concentrations and found that $50.4 \%$ of patients first measurement were low. The clinical efficacy of using drug levels to achieve adequate concentrations had never been properly evaluated. In a recent study, PD modelling was used to empirically treat 94 VAP in critically ill patients at high risk of infection with antibiotic-resistant Pseudomonas aeruginosa [81]. A three-hour infusion regimen of either cefepime or meropenem at a high dosage ( $2 \mathrm{~g}$ tid) was initiated, followed by both antibiotic and dose de-escalation whenever bacteria with a low MIC were identified. The infection-related mortality decreased from $21.6 \%$ to $8.5 \%(P=0.029)$.

The PD targets of $\beta$-lactam antibiotics may be different in patients with severe bacterial infections. McKinnon et al. [82] evaluated ceftazidime and cefepime PD by using PK data from previous clinical trials [83]. Maintaining a T > MIC as high as $100 \%$ was associated with a significantly greater clinical cure and bacteriologic eradication than a shorter time (cefatzidime: $82 \%$ vs. $33 \%, \mathrm{P}=0.002$; cefepime: $97 \%$ vs. $44 \%, \mathrm{P}=0.001$ ). Also, in a febrile neutropenia population of 60 patients treated with meropenem, a calculated T > MIC of $83 \%$ was found in responders, whilst those with a poor clinical response had a $\mathrm{T}>\mathrm{MIC}$ of only $60 \%$ [84]. It has also been suggested that, at least in vivo, maximum killing of bacteria is achieved at higher concentrations, four to five times MIC [85], accounting for antibiotic penetration in infected tissues. As such, concentrations of $\beta$-lactam antibiotics may need to be maintained well above the MIC for extended periods, especially in patients with life-threatening infections. Accordingly, different PD targets have been proposed in the different studies addressing ICU patients, which sometimes make their comparison difficult. 
An improved PD profile of $\beta$-lactams may be obtained by promoting a longer exposure with more frequent dosing, extended infusions or continuous infusions $[86,87]$. Several of the studies that we reviewed reported PD benefits of continuous infusions (even using small daily doses) (Table 2). Also, PD modelling tends to support this strategy. Nevertheless, almost none of the studies addressed reported a decrease in mortality. In addition, a recently published meta-analysis of 14 prospective studies did not show a significant benefit of using this strategy (odds ratio 1.00, 95\% confidence interval 0.48 to $2.06 ; P=1.00$ ) [88].

An increasing number of studies have addressed $\beta$-lactam antibiotic tissue concentration. Despite the theoretical advantage of analysing the drug concentration at the site of infection, there are no data to support a relationship between these concentrations and outcomes. Furthermore, there are still controversial issues involved in interpreting these data, namely, microdialysis [32,33]. Therefore, we think that, at present, no recommendation can be made regarding antibiotic tissue PK.

\section{Conclusions}

The PK of $\beta$-lactam antibiotics are significantly changed in septic ICU patients. Dosage and schedule regimens based on data from healthy volunteers may be misleading. Therapeutic drug monitoring and PD modelling according to measured PK previously showed promising results. Continuous infusion, although theoretically useful, has not been shown to lead to improved outcomes. The clinical significance of tissue PK monitoring remains to be determined.

\section{Key messages}

- Among ICU patients, the PK of $\beta$-lactam antibiotics are markedly unpredictable.

- A large volume of distribution is commonly observed in ICU patients and contributes to a lower antibiotic concentration, but also to a greater exposure time.

- An increased glomerular filtration rate is usually associated with a short half-life of $\beta$-lactam antibiotics, whilst renal failure is associated with a greater exposure and increased risk of accumulation.

- Continuous infusion of $\beta$-lactam antibiotics commonly increases the time that the antibiotic concentration exceeds its MIC and may therefore increase efficacy.

- Therapeutic drug monitoring of $\beta$-lactam antibiotic concentration may help to improve its efficacy and prevent toxicity, but currently is unavailable in most clinical settings.

\section{Abbreviations}

AUC: area under the concentration time curve; bid: dose every 12 hours; CFR: cumulative fraction of response; Cl: drug clearance; $\mathrm{Cr} \mathrm{Cl}$ : creatinine clearance; ELF: epithelial lining fluid; $f$ free drug fraction; $K_{e}$ : elimination rate constant; MIC: minimum inhibitory concentration; $\mathrm{MIC}_{90}$ : $90^{\text {th }}$ percentile of MIC in a bacteria population; PD: pharmacodynamics; PK: pharmacokinetics; PTA: probability of target attainment; qid: dose every six hours; $T_{1 / 2}$ : half-life; tid: dose every eight hours; $T>M I C$ : antibiotic concentration time over bacteria MIC; VAP: ventilator-associated pneumonia; $V_{\mathrm{d}}$ : volume of distribution

\section{Author details}

${ }^{1}$ Polyvalent Intensive Care Unit, São Francisco Xavier Hospital, Estrada do Forte do Alto do Duque, 1449-005 Lisboa, Portugal. ${ }^{2}$ CEDOC, Faculty of Medical Sciences, New University of Lisbon, Campo dos Mártires da Pátria, 130, 1169-056 Lisboa, Portugal.

\section{Authors' contributions}

Both JGP and PP searched the literature, analysed the data and wrote the manuscript. Both authors read and approved the final manuscript for publication.

\section{Competing interests}

JGP has received honoraria from and served as an advisor for Pfizer, AstraZeneca, Gilead Sciences Inc., Abbott Laboratories, Wyeth Lederle, Janssen-Cilag and Merck Sharp \& Dohme Corp. JGP also has received an unrestricted research grant from AstraZeneca. PP has received honoraria from and served as an advisor for AstraZeneca, Ely Lilly and Co., Gilead Sciences Inc., Janssen-Cilag, Merck Sharp \& Dohme Corp., Novartis and Pfizer Inc.

Received: 30 May 2011 Revised: 28 June 2011

Accepted: 13 September 2011 Published: 13 September 2011

\section{References}

1. Brun-Buisson C: The epidemiology of the systemic inflammatory response. Intensive Care Med 2000, 26(Suppl 1):S64-S74.

2. Vincent JL, Rello J, Marshall J, Silva E, Anzueto A, Martin CD, Moreno R, Lipman J, Gomersall C, Sakr Y, EPIC II Group of Investigators: International study of the prevalence and outcomes of infection in intensive care units. JAMA 2009, 302:2323-2329.

3. Póvoa P: Serum markers in community-acquired pneumonia and ventilator-associated pneumonia. Curr Opin Infect Dis 2008, 21:157-162.

4. Craig WA: Basic pharmacodynamics of antibacterials with clinical applications to the use of $\beta$-lactams, glycopeptides, and linezolid. Infect Dis Clin North Am 2003, 17:479-501.

5. Estes L: Review of pharmacokinetics and pharmacodynamics of antimicrobial agents. Mayo Clin Proc 1998, 73:1114-1122.

6. Pea F, Viale P, Furlanut M: Antimicrobial therapy in critically ill patients: a review of pathophysiological conditions responsible for altered disposition and pharmacokinetic variability. Clin Pharmacokinet 2005, 44:1009-1034.

7. Mehrotra R, De Gaudio R, Palazzo M: Antibiotic pharmacokinetic and pharmacodynamic considerations in critical illness. Intensive Care Med 2004, 30:2145-2156.

8. Fuster-Lluch O, Gerónimo-Pardo M, Peyró-García R, Lizán-García M: Glomerular hyperfiltration and albuminuria in critically ill patients. Anaesth Intensive Care 2008, 36:674-680.

9. Baptista JP, Udy AA, Sousa E, Pimentel J, Wang L, Roberts JA, Lipman J: A comparison of estimates of glomerular filtration in critically ill patients with augmented renal clearance. Crit Care 2011, 15:R139.

10. Verdant $C$, De Backer D: How monitoring of the microcirculation may help us at the bedside. Curr Opin Crit Care 2005, 11:240-244.

11. Levitt DG: The pharmacokinetics of the interstitial space in humans. BMC Clin Pharmacol 2003, 3:3.

12. Triginer C, Izquierdo I, Fernandez R, Rello J, Torrent J, Benito S, Net A: Gentamicin volume of distribution in critically ill septic patients. Intensive Care Med 1990, 16:303-306.

13. Hansen M, Christrup LL, Jarløv JO, Kampmann JP, Bonde J: Gentamicin dosing in critically ill patients. Acta Anaesthesiol Scand 2001, 45:734-740. 
14. Roberts JA, Lipman J: Antibacterial dosing in intensive care: pharmacokinetics, degree of disease and pharmacodynamics of sepsis. Clin Pharmacokinet 2006, 45:755-773.

15. Drusano GL: Pharmacokinetics and pharmacodynamics of antimicrobials. Clin Infect Dis 2007, 45(Suppl 1):S89-S95.

16. Mouton JW: Impact of pharmacodynamics on breakpoint selection for susceptibility testing. Infect Dis Clin North Am 2003, 17:579-598.

17. Choi G, Gomersall CD, Tian Q, Joynt GM, Li AM, Lipman J: Principles of antibacterial dosing in continuous renal replacement therapy. Blood Purif 2010, 30:195-212.

18. Shah PM: Parenteral carbapenems. Clin Microbiol Infect 2008, 14:175-180.

19. de Stoppelaar F, Stolk L, van Tiel F, Beysens A, van der Geest S, de Leeuw P: Meropenem pharmacokinetics and pharmacodynamics in patients with ventilator-associated pneumonia. J Antimicrob Chemother 2000, 46:150-151.

20. Jaruratanasirikul S, Sriwiriyajan S, Punyo J: Comparison of the pharmacodynamics of meropenem in patients with ventilator-associated pneumonia following administration by 3-hour infusion or bolus injection. Antimicrob Agents Chemother 2005, 49:1337-1339.

21. Kitzes-Cohen R, Farin D, Piva G, De Myttenaere-Bursztein SA: Pharmacokinetics and pharmacodynamics of meropenem in critically ill patients. Int J Antimicrob Agents 2002, 19:105-110.

22. Lovering AM, Vickery CJ, Watkin DS, Leaper D, McMullin CM, White LO, Reeves DS, MacGowan AP: The pharmacokinetics of meropenem in surgical patients with moderate or severe infections. J Antimicrob Chemother 1995, 36:165-172.

23. Taccone FS, Laterre PF, Dugernier $T$, Spapen $H$, Delattre I, Witebolle $X$, De Backer D, Layeux B, Wallemacq P, Vincent $J$, Jacobs F: Insufficient $\beta$ lactam concentrations in the early phase of severe sepsis and septic shock. Crit Care 2010, 14:R126.

24. Roberts JA, Kirkpatrick CM, Roberts MS, Robertson TA, Dalley AJ, Lipman J: Meropenem dosing in critically ill patients with sepsis and without renal dysfunction: intermittent bolus versus continuous administration? Monte Carlo dosing simulations and subcutaneous tissue distribution. J Antimicrob Chemother 2009, 64:142-150.

25. Karjagin J, Lefeuvre S, Oselin K, Kipper K, Marchand S, Tikkerberi A Starkopf J, Couet W, Sawchuk RJ: Pharmacokinetics of meropenem determined by microdialysis in the peritoneal fluid of patients with severe peritonitis associated with septic shock. Clin Pharmacol Ther 2008, 83:452-459.

26. Rhomberg PR, Jones RN: Contemporary activity of meropenem and comparator broad-spectrum agents: MYSTIC program report from the United States component (2005). Diagn Microbiol Infect Dis 2007, 57:207-215.

27. Thalhammer F, Traunmüller F, El Menyawi I, Frass M, Hollenstein UM, Locker GJ, Stoiser B, Staudinger T, Thalhammer-Scherrer R, Burgmann H: Continuous infusion versus intermittent administration of meropenem in critically ill patients. J Antimicrob Chemother 1999, 43:523-527.

28. Belzberg H, Zhu J, Cornwell EE, Murray JA, Sava J, Salim A, Velmahos GC, Gill MA: Imipenem levels are not predictable in the critically ill patient. J Trauma 2004, 56:111-117.

29. Novelli A, Adembri C, Livi P, Fallani S, Mazzei T, De Gaudio AR: Pharmacokinetic evaluation of meropenem and imipenem in critically ill patients with sepsis. Clin Pharmacokinet 2005, 44:539-549.

30. Jaruratanasirikul S, Sudsai T: Comparison of the pharmacodynamics of imipenem in patients with ventilator-associated pneumonia following administration by 2 or $0.5 \mathrm{~h}$ infusion. J Antimicrob Chemother 2009, 63:560-563.

31. Sakka SG, Glauner AK, Bulitta JB, Kinzig-Schippers M, Pfister W, Drusano GL, Sörgel F: Population pharmacokinetics and pharmacodynamics of continuous versus short-term infusion of imipenem-cilastatin in critically ill patients in a randomized, controlled trial. Antimicrob Agents Chemother 2007, 51:3304-3310.

32. Tegeder I, Schmidtko A, Bräutigam L, Kirschbaum A, Geisslinger G, Lötsch J: Tissue distribution of imipenem in critically ill patients. Clin Pharmacol Ther 2002, 71:325-333.

33. Dahyot C, Marchand S, Bodin M, Debeane B, Mimoz O, Couet W: Application of basic pharmacokinetic concepts to analysis of microdialysis data: illustration with imipenem muscle distribution. Clin Pharmacokinet 2008, 47:181-189.
34. McKindley DS, Boucher BA, Hess MM, Croce MA, Fabian TC Pharmacokinetics of aztreonam and imipenem in critically ill patients with pneumonia. Pharmacotherapy 1996, 16:924-931.

35. Muller-Serieys C, Bergogne-Berezin E, Rowan C, Dombret MC: Imipenem penetration into bronchial secretions. J Antimicrob Chemother 1987, 20:618-619.

36. Shikuma LR, Ackerman BH, Weaver RH, Solem LD, Strate RG, Cerra FB, Zaske DE: Effects of treatment and the metabolic response to injury on drug clearance: a prospective study with piperacillin. Crit Care Med 1990, 18:37-41.

37. Conil JM, Georges B, Mimoz O, Dieye E, Ruiz S, Cougot P, Samii K, Houin G, Saivin $S$ : Influence of renal function on trough serum concentrations of piperacillin in intensive care unit patients. Intensive Care Med 2006, 32:2063-2066.

38. Bourget $\mathrm{P}$, Lesne-Hulin $\mathrm{A}$, Le Reveillé $\mathrm{R}$, Le Bever $\mathrm{H}$, Carsin H: Clinical pharmacokinetics of piperacillin-tazobactam combination in patients with major burns and signs of infection. Antimicrob Agents Chemother 1996, 40:139-145.

39. Langgartner J, Lehn N, Glück T, Herzig H, Kees F: Comparison of the pharmacokinetics of piperacillin and sulbactam during intermittent and continuous intravenous infusion. Chemotherapy 2007, 53:370-377.

40. Rafati MR, Rouini MR, Mojtahedzadeh M, Najafi A, Tavakoli H, Gholami K, Fazeli MR: Clinical efficacy of continuous infusion of piperacillin compared with intermittent dosing in septic critically ill patients. Int J Antimicrob Agents 2006, 28:122-127.

41. Boselli E, Breilh D, Cannesson M, Xuereb F, Rimmelé T, Chassard D, Saux MC, Allaouchiche B: Steady-state plasma and intrapulmonary concentrations of piperacillin/tazobactam $4 \mathrm{~g} / 0.5 \mathrm{~g}$ administered to critically ill patients with severe nosocomial pneumonia. Intensive Care Med 2004, 30:976-979.

42. Jehl F, Muller-Serieys C, de Larminat V, Monteil H, Bergogne-Berezin E: Penetration of piperacillin-tazobactam into bronchial secretions after multiple doses to intensive care patients. Antimicrob Agents Chemother 1994, 38:2780-2784.

43. Boselli E, Breilh D, Rimmelé T, Guillaume C, Xuereb F, Saux MC, Bouvet $L$, Chassard D, Allaouchiche B: Alveolar concentrations of piperacillin/ tazobactam administered in continuous infusion to patients with ventilator-associated pneumonia. Crit Care Med 2008, 36:1500-1506.

44. Joukhadar C, Frossard M, Mayer BX, Brunner M, Klein N, Siostrzonek P, Eichler $H G$, Müller M: Impaired target site penetration of $\beta$-lactams may account for therapeutic failure in patients with septic shock. Crit Care Med 2001, 29:385-391.

45. Roberts JA, Roberts MS, Robertson TA, Dalley AJ, Lipman J: Piperacillin penetration into tissue of critically ill patients with sepsis: bolus versus continuous administration? Crit Care Med 2009, 37:926-933.

46. Roberts JA, Kirkpatrick CM, Roberts MS, Dalley AJ, Lipman J: First-dose and steady-state population pharmacokinetics and pharmacodynamics of piperacillin by continuous or intermittent dosing in critically ill patients with sepsis. Int J Antimicrob Agents 2009, 35:156-163.

47. Jacolot A, Incagnoli P, Edouard AR, Tod M, Petitjean O, Samii K, Mimoz O: Pharmacokinetics of cefpirome during the posttraumatic systemic inflammatory response syndrome. Intensive Care Med 1999, 25:486-491.

48. Lipman J, Wallis SC, Rickard CM, Fraenkel D: Low cefpirome levels during twice daily dosing in critically ill septic patients: pharmacokinetic modelling calls for more frequent dosing. Intensive Care Med 2001, 27:363-370.

49. Lipman J, Wallis SC, Boots RJ: Cefepime versus cefpirome: the importance of creatinine clearance. Anesth Analg 2003, 97:1149-1154.

50. Roos JF, Lipman J, Kirkpatrick CM: Population pharmacokinetics and pharmacodynamics of cefpirome in critically ill patients against Gramnegative bacteria. Intensive Care Med 2007, 33:781-788.

51. Sauermann R, Delle-Karth G, Marsik C, Steiner I, Zeitlinger M, MayerHelm BX, Georgopoulos A, Müller M, Joukhadar C: Pharmacokinetics and pharmacodynamics of cefpirome in subcutaneous adipose tissue of septic patients. Antimicrob Agents Chemother 2005, 49:650-655.

52. Joukhadar C, Klein N, Mayer BX, Kreischitz N, Delle-Karth G, Palkovits $P$, Heinz G, Müller M: Plasma and tissue pharmacokinetics of cefpirome in patients with sepsis. Crit Care Med 2002, 30:1478-1482.

53. Kieft H, Hoepelman Al, Knupp CA, van Dijk A, Branger JM, Struyvenberg A, Verhoef J: Pharmacokinetics of cefepime in patients with the sepsis syndrome. J Antimicrob Chemother 1993, 32(Suppl B):117-122. 
54. Conil JM, Georges B, Lavit M, Seguin T, Tack I, Samii K, Chabanon G, Houin G, Saivin S: Pharmacokinetics of ceftazidime and cefepime in burn patients: the importance of age and creatinine clearance. Int J Clin Pharmacol Ther 2007, 45:529-538.

55. Chapuis TM, Giannoni E, Majcherczyk PA, Chioléro R, Schaller MD, Berger MM, Bolay S, Décosterd LA, Bugnon D, Moreillon P: Prospective monitoring of cefepime in intensive care unit adult patients. Crit Care 2010, 14:R51.

56. Lipman J, Wallis SC, Rickard C: Low plasma cefepime levels in critically ill septic patients: pharmacokinetic modeling indicates improved troughs with revised dosing. Antimicrob Agents Chemother 1999, 43:2559-2561.

57. Bonapace $C R$, White RL, Friedrich LV, Norcross ED, Bosso JA: Pharmacokinetics of cefepime in patients with thermal burn injury. Antimicrob Agents Chemother 1999, 43:2848-2854.

58. Georges B, Conil JM, Seguin T, Dieye E, Cougot P, Decun JF, Lavit M, Samii K, Houin G, Saivin S: Cefepime in intensive care unit patients: validation of a population pharmacokinetic approach and influence of covariables. Int I Clin Pharmacol Ther 2008, 46:157-164

59. Nicasio AM, Ariano RE, Zelenitsky SA, Kim A, Crandon JL, Kuti J Nicolau DP: Population pharmacokinetics of high-dose, prolongedinfusion cefepime in adult critically ill patients with ventilator-associated pneumonia. Antimicrob Agents Chemother 2009, 53:1476-1481.

60. Roos JF, Bulitta J, Lipman J, Kirkpatrick CM: Pharmacokineticpharmacodynamic rationale for cefepime dosing regimens in intensive care units. J Antimicrob Chemother 2006, 58:987-993.

61. Sampol E, Jacquet A, Viggiano M, Bernini V, Manelli JC, Lacarelle B, Durand A: Plasma, urine and skin pharmacokinetics of cefepime in burns patients. J Antimicrob Chemother 2000, 46:315-317.

62. Klekner A, Bágyi K, Bognár L, Gáspár A, Andrási M, Szabó J: Effectiveness of cephalosporins in the sputum of patients with nosocomial bronchopneumonia. J Clin Microbiol 2006, 44:3418-3421.

63. Boselli E, Breilh D, Duflo F, Saux MC, Debon R, Chassard D, Allaouchiche B: Steady-state plasma and intrapulmonary concentrations of cefepime administered in continuous infusion in critically ill patients with severe nosocomial pneumonia. Crit Care Med 2003, 31:2102-2106.

64. Rondanelli R, Dionigi RV, Regazzi MB, Maurelli M, Calvi M, Mapelli A: Ceftazidime in the treatment of Pseudomonas infections in intensivecare patients. Int J Clin Pharmacol Ther Toxicol 1986, 24:457-459.

65. Young RJ, Lipman J, Gin T, Gomersall CD, Joynt GM, Oh TE: Intermittent bolus dosing of ceftazidime in critically ill patients. J Antimicrob Chemother 1997, 40:269-273.

66. Gómez CM, Cordingly JJ, Palazzo MG: Altered pharmacokinetics of ceftazidime in critically ill patients. Antimicrob Agents Chemother 1999, 43:1798-1802.

67. Benko AS, Cappelletty DM, Kruse JA, Rybak MJ: Continuous infusion versus intermittent administration of ceftazidime in critically ill patients with suspected gram-negative infections. Antimicrob Agents Chemother 1996, 40:691-695

68. Lipman J, Gomersall CD, Gin T, Joynt GM, Young RJ: Continuous infusion ceftazidime in intensive care: a randomized controlled trial. J Antimicrob Chemother 1999, 43:309-311.

69. Boselli E, Breilh D, Rimmelé T, Poupelin JC, Saux MC, Chassard D, Allaouchiche B: Plasma and lung concentrations of ceftazidime administered in continuous infusion to critically ill patients with severe nosocomial pneumonia. Intensive Care Med 2004, 30:989-991.

70. Hanes SD, Wood GC, Herring V, Croce MA, Fabian TC, Pritchard E, Boucher BA: Intermittent and continuous ceftazidime infusion for critically ill trauma patients. Am J Surg 2000, 179:436-440.

71. Angus BJ, Smith MD, Suputtamongkol Y, Mattie H, Walsh AL, Wuthiekanun V, Chaowagul W, White NJ: Pharmacokineticpharmacodynamic evaluation of ceftazidime continuous infusion vs intermittent bolus injection in septicaemic melioidosis. $\mathrm{Br} J \mathrm{Clin}$ Pharmacol 2000, 50:184-191.

72. Aubert G, Carricajo A, Coudrot M, Guyomarc'h S, Auboyer C, Zeni F: Prospective determination of serum ceftazidime concentrations in intensive care units. Ther Drug Monit 2010, 32:517-519.

73. Georges B, Conil JM, Seguin T, Ruiz S, Minville V, Cougot P, Decun JF, Gonzalez H, Houin G, Fourcade O, Saivin S: Population pharmacokinetics of ceftazidime in intensive care unit patients: influence of glomerular filtration rate, mechanical ventilation, and reason for admission. Antimicrob Agents Chemother 2009, 53:4483-4489.
74. Buijk SL, Gyssens IC, Mouton JW, Van Vliet A, Verbrugh HA, Bruining HA: Pharmacokinetics of ceftazidime in serum and peritoneal exudate during continuous versus intermittent administration to patients with severe intra-abdominal infections. J Antimicrob Chemother 2002, 49:121-128.

75. Mouton JW, Punt N, Vinks AA: A retrospective analysis using Monte Carlo simulation to evaluate recommended ceftazidime dosing regimens in healthy volunteers, patients with cystic fibrosis, and patients in the intensive care unit. Clin Ther 2005, 27:762-772.

76. Langer M, Cantoni P, Bellosta C, Boccazzi A: Penetration of ceftazidime into bronchial secretions in critically ill patients. J Antimicrob Chemother 1991, 28:925-932.

77. Bressolle F, de La Coussaye JE, Ayoub R, Fabre D, Gomeni R, Saissi G, Eledjam JJ, Galtier M: Endotracheal and aerosol administrations of ceftazidime in patients with nosocomial pneumonia: pharmacokinetics and absolute bioavailability. Antimicrob Agents Chemother 1992, 36:1404-1411.

78. Fridkin SK, Steward CD, Edwards JR, Pryor ER, McGowan JE Jr, Archibald LK, Gaynes RP, Tenover FC, Project Intensive Care Antimicrobial Resistance Epidemiology (ICARE) Hospitals: Surveillance of antimicrobial use and antimicrobial resistance in United States hospitals: Project ICARE Phase 2. Clin Infect Dis 1999, 29:245-252.

79. Chatellier D, Jourdain M, Mangalaboyi J, Ader F, Chopin C, Derambure P, Fourrier F: Cefepime-induced neurotoxicity: an underestimated complication of antibiotherapy in patients with acute renal failure. Intensive Care Med 2002, 28:214-217.

80. Roberts JA, Ulldemolins M, Roberts MS, McWhinney B, Ungerer J, Paterson DL, Lipman J: Therapeutic drug monitoring of $\beta$-lactams in critically ill patients: proof of concept. Int J Antimicrob Agents 2010, 36:332-339.

81. Nicasio AM, Eagye KJ, Nicolau DP, Shore E, Palter M, Pepe J, Kuti JL: Pharmacodynamic-based clinical pathway for empiric antibiotic choice in patients with ventilator-associated pneumonia. J Crit Care 2010, 25:69-77.

82. MCKinnon PS, Paladino JA, Schentag JJ: Evaluation of area under the inhibitory curve (AUIC) and time above the minimum inhibitory concentration ( $\mathrm{T}>\mathrm{MIC}$ ) as predictors of outcome for cefepime and ceftazidime in serious bacterial infections. Int J Antimicrob Agents 2008, 31:345-351.

83. Gentry LO, Rodriguez-Gomez G: Randomized comparison of cefepime and ceftazidime for treatment of skin, surgical wound, and complicated urinary tract infections in hospitalized subjects. Antimicrob Agents Chemother 1991, 35:2371-2374.

84. Ariano RE, Nyhlén A, Donnelly JP, Sitar DS, Harding GK, Zelenitsky SA: Pharmacokinetics and pharmacodynamics of meropenem in febrile neutropenic patients with bacteremia. Ann Pharmacother 2005, 39:32-38.

85. Mouton JW, Vinks AA, Punt NC: Pharmacokinetic-pharmacodynamic modeling of activity of ceftazidime during continuous and intermittent infusion. Antimicrob Agents Chemother 1997, 41:733-738.

86. Nicolau DP: Pharmacodynamic optimization of $\beta$-lactams in the patient care setting. Crit Care 2008, 12:S2.

87. Kasiakou SK, Sermaides GJ, Michalopoulos A, Soteriades ES, Falagas ME: Continuous versus intermittent intravenous administration of antibiotics: a meta-analysis of randomised controlled trials. Lancet Infect Dis 2005, 5:581-589.

88. Roberts JA, Webb S, Paterson D, Ho KM, Lipman J: A systematic review on clinical benefits of continuous administration of $\beta$-lactam antibiotics. Crit Care Med 2009, 37:2071-2078.

89. Ljungberg B, Nilsson-Ehle I: Pharmacokinetics of meropenem and its metabolite in young and elderly healthy men. Antimicrob Agents Chemother 1992, 36:1437-1440.

90. Drusano GL, Standiford HC, Bustamante C, Forrest A, Rivera G, Leslie J, Tatem B, Delaportas D, MacGregor RR, Schimpff SC: Multiple-dose pharmacokinetics of imipenem-cilastatin. Antimicrob Agents Chemother 1984, 26:715-721.

91. Paradis D, Vallée F, Allard S, Bisson C, Daviau N, Drapeau C, Auger F, LeBel M: Comparative study of pharmacokinetics and serum bactericidal activities of cefpirome, ceftazidime, ceftriaxone, imipenem, and ciprofloxacin. Antimicrob Agents Chemother 1992, 36:2085-2092.

92. Barbhaiya RH, Forgue ST, Gleason CR, Knupp CA, Pittman KA, Weidler DJ, Movahhed H, Tenney J, Martin RR: Pharmacokinetics of cefepime after 
single and multiple intravenous administrations in healthy subjects. Antimicrob Agents Chemother 1992, 36:552-557.

93. Dawson-Saunders B, Trapp RG: Basic \& Clinical Biostatistics. 2 edition. Chicago: Appleton \& Lange; 1994.

doi:10.1186/cc10441

Cite this article as: Gonçalves-Pereira and Póvoa: Antibiotics in critically ill patients: a systematic review of the pharmacokinetics of $\beta$-lactams. Critical Care 2011 15:R206.

Submit your next manuscript to BioMed Central and take full advantage of:

- Convenient online submission

- Thorough peer review

- No space constraints or color figure charges

- Immediate publication on acceptance

- Inclusion in PubMed, CAS, Scopus and Google Scholar

- Research which is freely available for redistribution

Submit your manuscript at www.biomedcentral.com/submit
() Biomed Central 